\title{
Co-estimating geomagnetic field and calibration parameters: modeling Earth's magnetic field with platform magnetometer data
}

\author{
Clemens Kloss ${ }^{*} \mathbb{D}$, Christopher C. Finlay and Nils Olsen
}

\begin{abstract}
Models of the geomagnetic field rely on magnetic data of high spatial and temporal resolution to give an accurate picture of the Earth's internal magnetic field and its time-dependence. The magnetic data from low-Earth orbit satellites of dedicated magnetic survey missions such as CHAMP and Swarm play a key role in the construction of such models. Unfortunately, there are no magnetic data available from such satellites after the end of the CHAMP mission in 2010 and before the launch of the Swarm mission in late 2013. This limits our ability to recover signals on timescales of 3 years and less during this gap period. The magnetic data from platform magnetometers carried by satellites for navigational purposes may help address this data gap provided that they are carefully calibrated. Earlier studies have demonstrated that platform magnetometer data can be calibrated using a fixed geomagnetic field model as reference. However, this approach can lead to biased calibration parameters. An alternative approach has been developed in the form of a co-estimation scheme which consists of simultaneously estimating both the calibration parameters and a model of the internal part of the geomagnetic field. Here, we go further and develop a scheme, based on the CHAOS field modeling framework, that involves co-estimation of both internal and external geomagnetic field models along with calibration parameters of platform magnetometer data. Using our implementation, we are able to derive a geomagnetic field model spanning 2008 to 2018 with satellite magnetic data from CHAMP, Swarm, secular variation data from ground observatories, and platform magnetometer data from CryoSat-2 and the GRACE satellite pair. Through a number of experiments, we explore correlations between the estimates of the geomagnetic field and the calibration parameters, and suggest how these may be avoided. We find evidence that platform magnetometer data provide additional information on the secular acceleration, especially in the Pacific during the gap between CHAMP and Swarm. This study adds to the evidence that it is beneficial to use platform magnetometer data in geomagnetic field modeling.
\end{abstract}

Keywords: Geomagnetism, Core field modeling, Inverse theory, Secular acceleration, Secular variation

\section{Introduction}

The Earth's magnetic field is a superposition of many sources. By far, the largest contribution comes from within the Earth at a depth of more than $3000 \mathrm{~km}$. There,

*Correspondence: ancklo@space.dtu.dk

Division of Geomagnetism, DTU Space, Technical University of Denmark, Centrifugevej 356, 2800 Kongens Lyngby, Denmark in the outer core, a liquid iron alloy is rapidly moving and thus advecting, stretching, and maintaining the ambient magnetic field against dissipation in a process called the Geodynamo. Earth's core dynamics are not fully understood, but can be studied using time-dependent geomagnetic field models. Such models are constructed using measurements of the magnetic field taken at and above Earth's surface. 
The study of core processes on decadal or longer timescales requires long time-series of magnetic vector data with high spatial and temporal resolution. Along with ground-based magnetic observatories, low-earth orbit satellites from dedicated magnetic survey missions such as CHAllenging Minisatellite Payload (CHAMP, 20002010) and the Swarm trio (since 2013) provide such data. However, other than scalar data from Ørsted, no highquality calibrated magnetic vector data from satellites are available between the end of the CHAMP mission in September 2010 and the launch of the Swarm satellites in November 2013. This data gap not only cuts in two an otherwise uninterrupted time-series of high-quality magnetic satellite data since the year 2000, but also limits our ability to derive accurate core field models that resolve temporal changes of the magnetic field on timescales of a few years and less in the gap period. To address the issue, one can utilize the crude magnetometers that are carried by most satellites for navigational purposes, the so-called platform magnetometers. Although not a substitute for dedicated high-quality magnetic survey satellites, platform magnetometers can supplement ground observatory data in gaps between dedicated missions and help improve the local time data coverage of simultaneously flying high-quality magnetic survey satellites.

Satellite-based magnetic vector data need to be calibrated to remove magnetometer biases, scale factors, and non-orthogonalities between the three vector component axes (Olsen et al. 2003). Comparing the vector magnetometer output with a magnetic reference field allows the estimation of these calibration parameters. On dedicated survey mission satellites, the reference is a second, absolute scalar, magnetometer mounted in close proximity to the vector magnetometer and measuring the magnetic field intensity. However, non-dedicated satellites carrying platform magnetometers are typically not equipped with such scalar reference magnetometers. In this case, it is possible to use a-priori geomagnetic field models like CHAOS (Olsen et al. 2006; Finlay et al. 2020) or the IGRF (Thébault et al. 2015) as reference. Such an approach has been successfully used, e.g., by Olsen et al. (2020) for calibrating data from the CryoSat-2 magnetometer, but use of a fixed reference field model is not without risks and could lead to biased calibration parameters.

An alternative venue has been explored by Alken et al. (2020), who combined high-quality magnetic data from CHAMP and Swarm with platform magnetometer data from CryoSat-2 and several satellites of the Defense Meteorological Satellite Program (DMSP) to estimate a model of the internal field and the required calibration parameters for each satellite simultaneously. Ideally, such a co-estimation scheme eliminates the need for a-priori geomagnetic field models, but Alken et al. (2020) fall short by co-estimating only the internal field while still relying on a fixed model of the external field. Nevertheless, their study convincingly demonstrated that platform magnetometer data provide valuable information about the time-dependence of Earth's magnetic field.

In this study, we followed Alken et al. (2020) and developed a co-estimation strategy but within the framework of the CHAOS field model series. Our implementation differs in three important aspects. First, we estimated both the internal (core and crust) and external (magnetospheric) geomagnetic field contributions in contrast to only the internal field. This way, we avoided having to remove a fixed external field model from the satellite data prior to the model parameter estimation. Following the methodology of the CHAOS model, we did use a prior external field model for processing the ground observatory data which we used in addition to the satellite data. Second, we used the platform magnetometer data from CryoSat-2 and, instead of DMSP, data from the Gravity Recovery and Climate Experiment (GRACE) satellite pair. Finally, to reduce the significant correlation between the internal axial dipole and the calibration parameters during periods of poor coverage of high-quality magnetic data, we excluded platform magnetometer data from determining the internal axial dipole (its time variation is well resolved with ground observatory data during the gap period, while its absolute value is constrained by Swarm and CHAMP data on both sides of the gap) rather than controlling the temporal variability of the internal axial dipole through an additional regularization as done by Alken et al. (2020).

The paper is organized as follows. In the first part, we present the datasets and the data processing. Next, we describe the model parameterization and define the calibration parameters, which are similar to those used for the Ørsted satellite (Olsen et al. 2003). We go on by presenting a geomagnetic field model derived from highquality calibrated data from the CHAMP and the Swarm satellites as well as ground observatory secular variation data and supplemented this with previously uncalibrated platform magnetometer data from CryoSat-2 and GRACE, spanning a 10 year period from 2008 to 2018 . Finally, we explore in a series of experiments the effect of co-estimating an external field, the trade-off between the internal dipole and the calibration parameters, and the importance of including dayside platform magnetometer data when estimating calibration parameters. We conclude the paper by looking at the secular acceleration of our model, paying particular attention to the data gap between 2010 and 2013. 


\section{Data and data processing}

We used calibrated magnetic data from the Swarm satellites Alpha (Swarm-A) and Bravo (Swarm-B), and from the CHAMP satellite from January 2008 to the end of December 2017, supplemented with five datasets of uncalibrated magnetic data from the three platform fluxgate magnetometers (FGM) on-board the CryoSat-2 satellite (CryoSat-2 FGM1, CryoSat-2 FGM2 and CryoSat-2 FGM3), the one on-board the first GRACE satellite (GRACE-A), and the other one on-board the second GRACE satellite (GRACE-B). In addition to the satellite data, we included revised monthly mean values of the SV from ground observatories to contribute to the Earth's internal time-dependent field. Details of the datasets are given in the following.

\section{Absolute satellite data from scientific magnetometers}

The satellite data from scientific magnetometers are in general of high quality in terms of accuracy, precision, and magnetic cleanliness. The high standard of the data is achieved by low-noise instruments that are mounted together with star cameras on an optical bench further away from the spacecraft body at the center of a several meter long boom. The data are regularly calibrated inflight with a second absolute scalar magnetometer placed at the end of the boom and carefully cleaned from magnetic disturbance fields originating from the spacecraft body.

From the CHAMP mission, we used the Level $31 \mathrm{~Hz}$ magnetic data, version CH-ME-3-MAG (Rother and Michaelis 2019), between January 2008 and August 2010, downsampled to $15 \mathrm{~s}$, and only when attitude information from both star cameras was available. From the Swarm mission, we used the Level $1 \mathrm{~b} 1 \mathrm{~Hz}$ magnetic data product, baseline 0505/0506, from the Swarm-A and SwarmB satellites between November 2013 and December 2018, also downsampled to $15 \mathrm{~s}$. Here, we worked with vector data from CHAMP and Swarm in the magnetometer frame.

\section{Relative satellite data from platform magnetometers}

Relative satellite data refer to the raw sensor output from platform magnetometers. The data have to be corrected and calibrated before they can be used in geomagnetic field modeling. The correction of the data accounts for temperature effects, magnetic disturbances due to solar array and battery currents, magnetorquer activity, as well as non-linear sensor effects, whereas the calibration removes magnetometer biases, scale differences, and non-orthogonalities between the three vector component axes.

From CryoSat-2, we took magnetic data, baseline 0103, from the three platform magnetometers as described in
Olsen et al. (2020) from August 2010 to December 2018 and only when the attitude uncertainty $q_{\text {error }}$ was below $40^{\prime \prime}$. Since the purpose of this paper is the co-estimation of calibration parameters for the platform magnetometers, we processed the dataset using the original calibration parameters to undo the calibration step that has been performed by Olsen et al. (2020) but keeping the applied correction for magnetic disturbances from the spacecraft and its payload. This way, we obtained essentially uncalibrated data while still retaining the corrections for magnetic disturbances, temperature effects and non-linearities. In a pre-whitening and data reduction step, we computed residuals to the CHAOS-6- $x$ 9 model in the uncalibrated magnetometer frame, removed those larger than $1000 \mathrm{eu}$ (quasi nanoTesla, in the following referred to as engineering units) in absolute value to discard gross outliers, computed component-wise robust mean values of the residuals in $1 \mathrm{~min}$ bins to reduce the original $4 \mathrm{~s}$ sampled data to $1 \mathrm{~min}$ values, and added the CHAOS-6- $x 9$ model values back. Figure 1 shows an example of the raw vector residuals $\Delta \mathbf{E}$ of CryoSat-2 FGM1 in the uncalibrated magnetometer frame over $3 \mathrm{~h}$ on March 24, 2016.

In a similar way, we processed the $1 \mathrm{~Hz}$ data from the GRACE satellites, baseline 0101, to obtain $1 \mathrm{~min}$ uncalibrated but corrected vector data between January 2008 and October 2017 (GRACE-A) and August 2017 (GRACE-B) (Olsen 2020).

The computation of $1 \mathrm{~min}$ values served two purposes. First, to reduce the random noise of the magnetometers by taking the average of successive values and, second, to decrease the number of platform magnetometer data, so that a fair amount of absolute satellite data was able to guide the co-estimation of the calibration parameters.

\section{Ground observatory data}

In addition to satellite data, we added annual differences of monthly mean values from 162 ground observatories to help determine the time changes of the core field (secular variation). Following Olsen et al. (2014), we computed revised monthly means as Huber-weighted averages of the hourly observatory mean values from the AUX OBS database (Macmillan and Olsen 2013) at all local times after removing estimates of the ionospheric field of the CM4 model (Sabaka et al. 2004) and the largescale magnetospheric field of CHAOS-6- 99 , including their internally induced parts.

\section{Satellite data selection}

We organized the satellite data according to quasi-dipole (QD) latitude (Richmond 1995) into a non-polar (equal to and equatorward of $\pm 55^{\circ}$ ) and a polar (poleward of $\left.\pm 55^{\circ}\right)$ data subset. From each subset, we selected data 


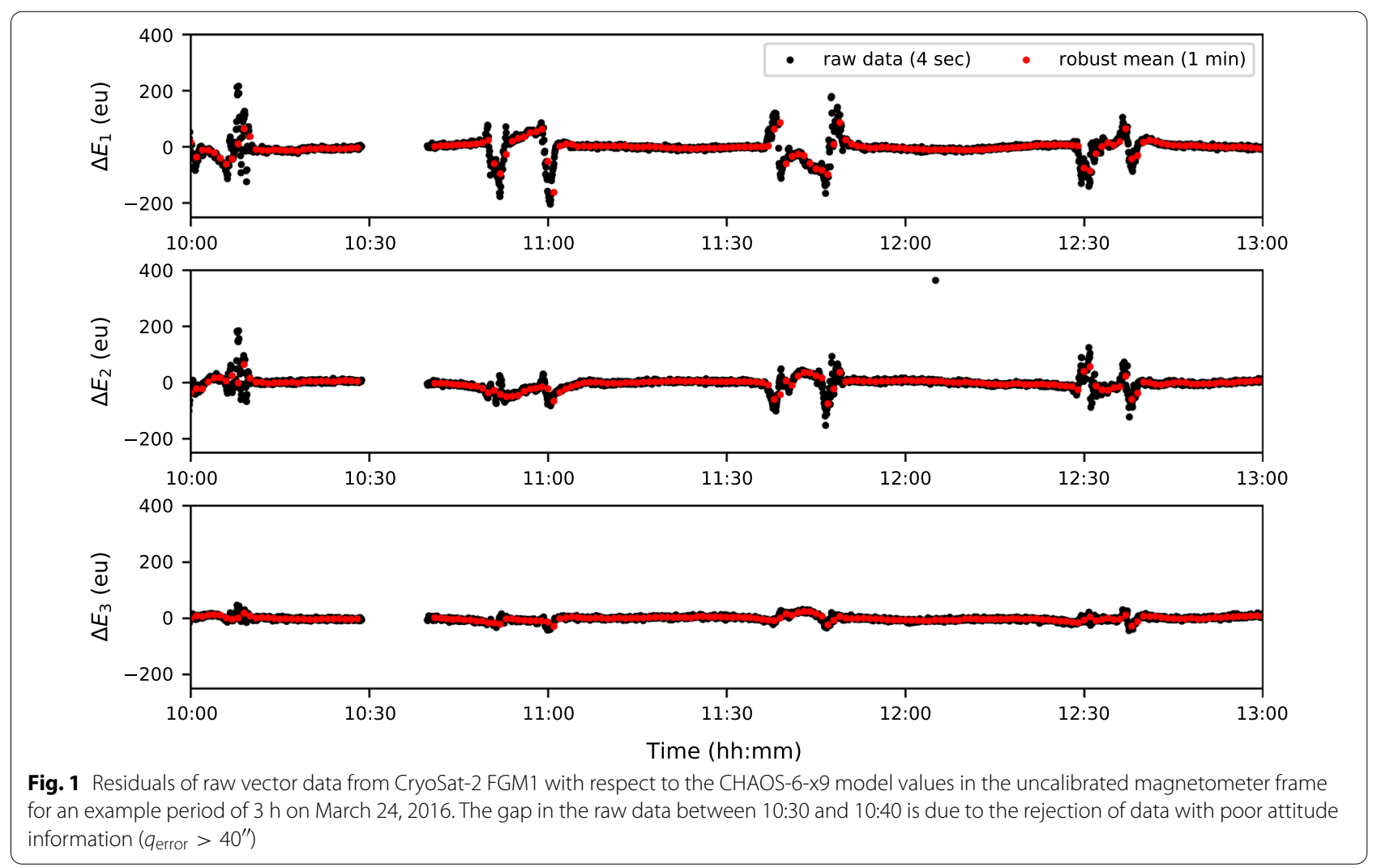

under quiet geomagnetic conditions. Specifically, we selected data from the non-polar subset that satisfied the following criteria:

- Low geomagnetic activity as indicated by the planetary activity index Kp smaller than or equal to $2^{o}$;

- Dark condition as indicated by a solar zenith angle greater than $100^{\circ}$ for the Swarm and CHAMP satellites (i.e., sun at least $10^{\circ}$ below the horizon). From CryoSat- 2 and GRACE, we used data from dark and sunlit regions, since we found that this leads to better determined calibration parameters;

- Slow change of the magnetospheric ring current as indicated by the RC-index (Olsen et al. 2014) rate of change in absolute terms being smaller than $2 \mathrm{nTh}^{-1}$.

From the polar subset, we kept data according to the following criteria:

- Dark condition except in the case of platform magnetometers on-board CryoSat-2 and GRACE, where we also used sunlit data;

- RC-index rate of change in absolute terms smaller than or equal to $2 \mathrm{nTh}^{-1}$;
- The merging electric field at the magnetopause $E_{\mathrm{m}}=v^{4 / 3} B_{\mathrm{T}}^{2 / 3} \sin |\Theta| / 2$, where $v$ is the solar wind speed, $B_{\mathrm{T}}=\sqrt{B_{y}^{2}+B_{z}^{2}}$ is the interplanetary magnetic field in the $y-z$-plane of the Geocentric Solar Magnetic (GSM) coordinates, and $\Theta=\arctan \left(B_{y} / B_{z}\right)$, was on average smaller than $2.4 \mathrm{mVm}^{-1}$ over the previous $2 \mathrm{~h}$;

- The interplanetary magnetic field component $B_{z}$ in GSM coordinates was on average positive over the previous $2 \mathrm{~h}$.

Figure 2 shows a stacked histogram of the number of data for each satellite after the data selection.

It can be clearly seen that platform magnetometer data are the main contributor to the number of data in the gap period, whereas it is comparable to the number of data from CHAMP and the Swarm satellites in the time before and after the gap. The ground observatories contribute approximately 130 monthly mean values of the SV each month throughout the entire model time span, which is much less than the monthly average number of satellite data. 


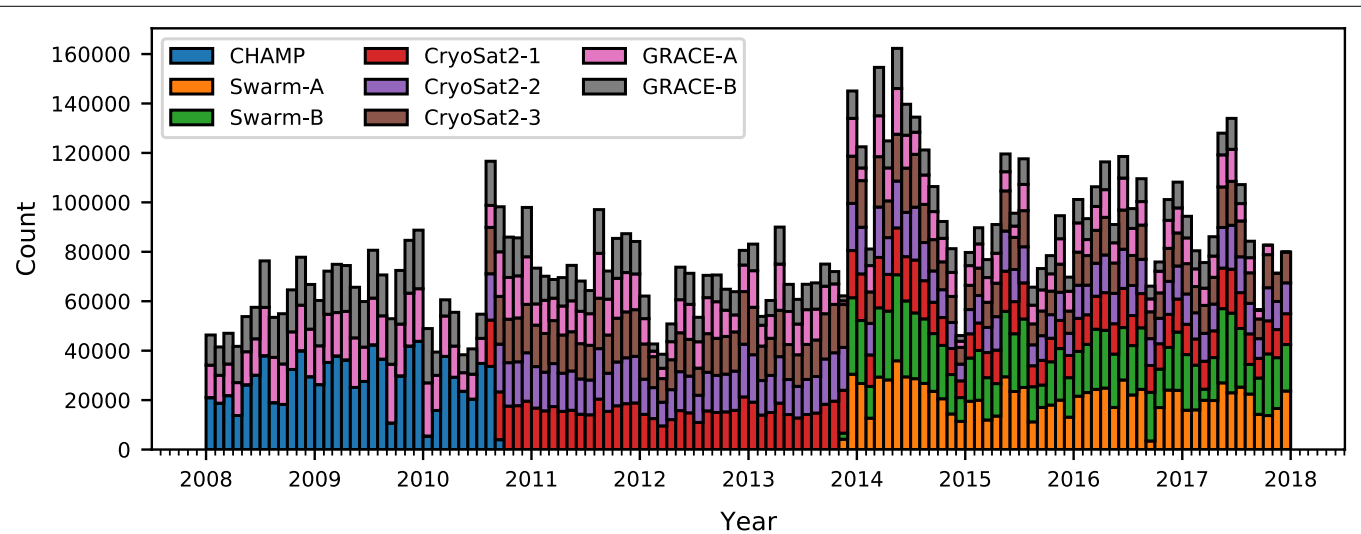

Fig. 2 Number of selected satellite data per month as stacked histogram. Ground observatories contribute with approximately 130 vector measurements of the SV per month.

\section{Model parameterization and estimation}

We are interested in the magnetic field vector $\mathbf{B}$ on length scales smaller than Earth's circumference and time scales that are much longer than the time it takes light to traverse these distances (Backus et al. 1996; Sabaka et al. 2010). On these scales, the displacement current can be neglected and the magnetic field is governed by Ampere's law. We assume that the measurements of Earth's magnetic field are taken in a region free of electrical currents and magnetized material, such that the field is irrotational, which allows us to introduce a scalar potential $V$ to represent the magnetic field as the gradient of the potential $\mathbf{B}=-\nabla V$. The potential consists of two terms $V=V_{\text {int }}+V_{\text {ext }}$ that describe internal sources such as the time-dependent core-generated field and the assumed static lithospheric field, and external sources that we assume are mainly magnetospheric in origin for our chosen data selection criteria and have an internally induced counterpart associated with them (by selecting data from dark regions, we minimize ionospheric field contributions).

To describe the geomagnetic field, we use an Earthfixed frame of reference whose point of origin coincides with the Earth's center and in which the position vector $\mathbf{r}$ is given in spherical polar coordinates by the radial distance $r$ as measured from the origin (radius), the angular distance $\theta$ (co-latitude) as measured from the north polar axis, and the azimuthal angular distance $\phi$ (longitude) as measured from the Greenwich meridian. In the following, we refer to that system as the Radius-Theta-Phi (RTP) reference frame.

In spherical coordinates, the scalar potential can be expressed as a weighted sum of solid harmonics, which are harmonic functions of the spatial coordinates. Our modeling approach follows that of earlier models of the
CHAOS model series (Olsen et al. 2006, 2014; Finlay et al. 2016, 2020) and consists of describing the geomagnetic field with the help of a scalar potential whose exact form depends on a set of coefficients that multiply the solid harmonics. The coefficients are estimated by minimizing a quadratic cost function in the residuals, which are the difference between the magnetic observations and the magnetic data calculated with the model. We used two kinds of residuals: the components of vector differences in the RTP frame (vector residuals) and the difference of vector magnitudes (scalar residuals). More specifically, we computed vector residuals of the nonpolar satellite data, scalar residuals of the polar satellite data, and vector residuals of the ground observatory SV data at all QD latitudes.

\section{Internal field parameters}

The scalar potential of the internal sources is given by:

$$
\begin{aligned}
V_{\text {int }}(\mathbf{r}, t)= & a \sum_{n=1}^{N_{\text {int }}} \sum_{m=0}^{n}\left(g_{n}^{m}(t) \cos m \phi\right. \\
& \left.+h_{n}^{m}(t) \sin m \phi\right)\left(\frac{a}{r}\right)^{n+1} P_{n}^{m}(\cos \theta),
\end{aligned}
$$

where $a=6371.2 \mathrm{~km}$ is the chosen spherical reference radius of the Earth, $n$ and $m$ are, respectively, the spherical harmonic degree and order, $N_{\text {int }}$ is the truncation degree, $g_{n}^{m}(t)$ and $h_{n}^{m}(t)$ are the Gauss coefficients in nanoTesla (nT) for a given $n$ and $m$, and $P_{n}^{m}(\cos \theta)$ are the Schmidt quasi-normalized associated Legendre functions. We truncated the formally infinite sum of solid harmonics at $N_{\text {int }}=50$ and expanded the Gauss coefficients of degree $n \leq 15$ in time using sixth-order B-splines (De Boor 1978), while we kept the higher degree coefficients $(n>15)$ constant in time: 


$$
g_{n}^{m}(t)=\left\{\begin{array}{l}
\sum_{j} g_{n, j}^{m} B_{6, j}(t), \quad n \leq 15 \\
g_{n}^{m}, \quad n>15,
\end{array}\right.
$$

where $g_{n, j}^{m}$ (similarly for $h_{n, j}^{m}$ ) is the coefficient of $B_{6, j}(t)$ -the $j$ th function of the B-spline basis that has knots at 6-month intervals and six-fold multiplicity at the model endpoints in $t_{\mathrm{s}}=2008.0$ and $t_{\mathrm{e}}=2018.0$ in years. For the purposes of testing the co-estimation of calibration parameters here, a truncation of the time-dependent internal field at degree $N_{\text {int }}=15$ was deemed sufficient.

\section{External field parameters}

The scalar potential of the external sources consists of two terms $V_{\text {ext }}=V_{\mathrm{SM}}+V_{\mathrm{GSM}}$ that are designed to account for near and remote magnetospheric sources. We use the Solar Magnetic (SM) coordinate system to parameterize near magnetospheric sources: bins of 30 days except in the gap period, where we used a single bin from August 2010 to January 2014 to reduce the strong co-linearity between the calibration parameters and the baseline corrections that earlier tests had revealed.

The remote magnetospheric sources, and the currents at the magnetopause and in the magnetotail, are taken into account by a purely zonal potential in the GSM coordinate system up to degree 2 :

$$
V_{\mathrm{GSM}}(\mathbf{r}, t)=a \sum_{n=1}^{2} q_{n, \mathrm{GSM}}^{0} R_{n, \mathrm{GSM}}^{0, \mathrm{c}}(\mathbf{r}, t),
$$

where $q_{n, \mathrm{GSM}}^{m}$ and $s_{n, \mathrm{GSM}}^{m}$ are Gauss coefficients that are constant in time with respect to the GSM coordinate system, and $R_{n, \mathrm{GSM}}^{m, \mathrm{c}}$ are modifications of the solid harmonics similar to corresponding terms in Eq. (3) but for the GSM coordinates.

$$
\begin{aligned}
V_{\mathrm{SM}} & =a \sum_{m=0}^{1}\left(q_{1, \mathrm{SM}}^{m}(r, t) \cos m \phi_{\mathrm{SM}}+s_{1, \mathrm{SM}}^{m}(r, t) \sin m \phi_{\mathrm{SM}}\right) P_{1}^{m}\left(\cos \theta_{\mathrm{SM}}\right) \\
& +a \sum_{m=0}^{1}\left(\Delta q_{1, \mathrm{SM}}^{m}(t) R_{1, \mathrm{SM}}^{m, \mathrm{c}}(\mathbf{r}, t)+\Delta s_{1, \mathrm{SM}}^{m}(t) R_{1, \mathrm{SM}}^{m, \mathrm{~s}}(\mathbf{r}, t)\right) \\
& +a \sum_{m=0}^{2}\left(q_{2, \mathrm{SM}}^{m, \mathrm{c}} R_{2, \mathrm{SM}}^{m, \mathrm{c}}(\mathbf{r}, t)+s_{2, \mathrm{SM}}^{m} R_{2, \mathrm{SM}}^{m, \mathrm{~s}}(\mathbf{r}, t)\right),
\end{aligned}
$$

where $\theta_{\mathrm{SM}}$ and $\phi_{\mathrm{SM}}$ are, respectively, the SM co-latitude and longitude, $q_{n, \mathrm{SM}}^{m}$ and $s_{n, \mathrm{SM}}^{m}$ are the Gauss coefficients with respect to the SM coordinate system, $\Delta q_{1, \mathrm{SM}}^{m}(t)$ and $\Delta s_{1, \mathrm{SM}}^{m}(t)$ are the RC-baseline corrections, and $R_{n, \mathrm{SM}}^{m, \mathrm{~s}}$ and $R_{n, \mathrm{SM}}^{m, \mathrm{c}}$ are modification of the solid harmonics that account for the time-dependent transformation from the SM to the geographic coordinate system and include internally induced contributions based on the diagonal part of the Q-response matrix that has been derived from a 3D conductivity model of Earth (Finlay et al. 2020). The external Gauss coefficients with $n=1$ have a specific time-dependence in the form of:

$$
\begin{aligned}
& q_{1, \mathrm{SM}}^{0}(r, t)=\hat{q}_{1}^{0}\left[\epsilon(t)\left(\frac{r}{a}\right)+\iota(t)\left(\frac{a}{r}\right)^{2}\right] \\
& q_{1, \mathrm{SM}}^{1}(r, t)=\hat{q}_{1}^{1}\left[\epsilon(t)\left(\frac{r}{a}\right)+\iota(t)\left(\frac{a}{r}\right)^{2}\right] \\
& s_{1, \mathrm{SM}}^{1}(r, t)=\hat{s}_{1}^{1}\left[\epsilon(t)\left(\frac{r}{a}\right)+\iota(t)\left(\frac{a}{r}\right)^{2}\right],
\end{aligned}
$$

where $\epsilon(t)$ and $\iota(t)$ are the respective internal and external part of the RC-index linearly interpolated from hourly values. The $\mathrm{RC}$-baseline corrections were estimated in

\section{Alignment parameters}

Using satellite data in the vector field magnetometer frame (VFM) requires an additional step, called data alignment, which involves determining alignment parameters that describe the rotation of the magnetic field vector $\mathbf{B}_{\mathrm{VFM}}$ in the VFM frame to $\mathbf{B}_{\mathrm{CRF}}$ in the common reference frame (CRF) of the satellite. Once in the $\mathrm{CRF}$, the vector components can be combined with the attitude information from the star camera and rotated into the RTP frame for computing the vector residuals. We performed the data alignment for CHAMP, Swarm, CryoSat-2, and GRACE.

The alignment parameters are usually parameterized in the form of Euler angles $\alpha, \beta$, and $\gamma$. We adopted the 1-2-3 convention of the Euler angles to align the magnetic field:

$$
\begin{aligned}
\mathbf{B}_{\mathrm{CRF}} & =\underline{\underline{\overline{\mathrm{R}}}} \mathrm{VFM}(\alpha, \beta, \gamma) \mathbf{B}_{\mathrm{VFM}} \\
& =\underline{\underline{\mathbf{R}_{3}}}(\gamma) \underline{\underline{\mathbf{R}_{2}}}(\beta) \underline{\underline{\mathbf{R}_{1}}}(\alpha) \mathbf{B}_{\mathrm{VFM}},
\end{aligned}
$$

where the rotation matrix is a combination of the three rotations: 


$$
\begin{aligned}
& \underline{\underline{\mathbf{R}_{1}}}=\left(\begin{array}{ccc}
1 & 0 & 0 \\
0 & \cos \alpha & -\sin \alpha \\
0 & \sin \alpha & \cos \alpha
\end{array}\right) \\
& \underline{\underline{\mathbf{R}_{2}}}=\left(\begin{array}{ccc}
\cos \beta & 0 & \sin \beta \\
0 & 1 & 0 \\
-\sin \beta & 0 & \cos \beta
\end{array}\right) \\
& \underline{\underline{\mathbf{R}_{3}}}=\left(\begin{array}{ccc}
\cos \gamma & -\sin \gamma & 0 \\
\sin \gamma & \cos \gamma & 0 \\
0 & 0 & 1
\end{array}\right) .
\end{aligned}
$$

Following the alignment, we applied another rotation matrix $\mathbf{R}_{\mathrm{RTP}}^{\mathrm{CRF}}$ to rotate the field components from the CRF to the RTP reference frame:

$$
\mathbf{B}_{\mathrm{RTP}}=\underline{\underline{\mathbf{R}_{\mathrm{RTP}}^{\mathrm{CRF}}}}(\mathbf{r}, t) \mathbf{B}_{\mathrm{CRF}},
$$

which depends on position and time. That rotation matrix was computed by combining the quaternions that express the rotation from the CRF to the Earth-fixed Earth-centered North-East-Center (NEC) frame with quaternions that describe the change from the NEC to the RTP reference frame. For each satellite dataset, we parameterized the Euler angles in time as a piecewise constant function using a sequence of 30 day bins.

\section{Calibration parameters}

The calibration can be viewed as an extension of the data alignment which makes it possible to use platform magnetometer data in geomagnetic field modeling. We performed the calibration for CryoSat-2 and the GRACE satellites.

We assume that the platform magnetometer is a linear vector field magnetometer, which provides information about the desired local magnetic field vector $\mathbf{B}_{\mathrm{VFM}}$ (units of nT) in the form of the sensor output $\mathbf{E}=\left(E_{1}, E_{2}, E_{3}\right)^{\mathrm{T}}$ (units of eu), which typically consists of components that are measured relative to three biased and non-orthogonal axes employing different scale factors (Olsen et al. 2003). More specifically, the sensor output in the magnetometer frame is related to the local magnetic field through:

$$
\mathbf{B}_{\mathrm{VFM}}=\underline{\underline{\mathbf{P}}}^{-1} \underline{\underline{\mathbf{S}}}^{-1}(\mathbf{E}-\mathbf{b}) \text {, }
$$

where

$$
\underline{\underline{\mathbf{S}}}(\mathbf{s})=\left(\begin{array}{ccc}
s_{1} & 0 & 0 \\
0 & s_{2} & 0 \\
0 & 0 & s_{3}
\end{array}\right)
$$

is the diagonal matrix of sensitivities or scale factors $\mathbf{s}=\left(s_{1}, s_{2}, s_{3}\right)^{\mathrm{T}}$ (units of eu/nT):

$$
\underline{\underline{\mathbf{P}}}(\mathbf{u})=\left(\begin{array}{ccc}
1 & 0 & 0 \\
-\sin u_{1} & \cos u_{1} & 0 \\
\sin u_{2} & \sin u_{3} & \sqrt{1-\sin ^{2} u_{2}-\sin ^{2} u_{3}}
\end{array}\right)
$$

is the matrix that projects the orthogonal components of magnetic field vector $\mathbf{B}_{\mathrm{VFM}}$ onto three non-orthogonal directions defined by the non-orthogonality angles $\mathbf{u}=\left(u_{1}, u_{2}, u_{3}\right)^{\mathrm{T}}$ (units of radians), and:

$$
\mathbf{b}=\left(\begin{array}{l}
b_{1} \\
b_{2} \\
b_{3}
\end{array}\right)
$$

is the offset or bias vector (units of eu). Combining the calibration step in Eq. (9), the alignment step involving the Euler angles in Eq. (6) and the change of frame in Eq. (8), yields an equation that transforms the uncalibrated sensor output $\mathbf{E}$ into calibrated, aligned field components in the RTP frame:

$$
\mathbf{B}_{\mathrm{RTP}}=\underline{\underline{\mathbf{R}_{\mathrm{RTP}}^{\mathrm{CR}}}}(r, \theta, \phi) \underline{\underline{\mathrm{CRF}}}(\alpha, \beta, \gamma) \underline{\underline{\mathbf{P}}}^{-1} \underline{\underline{\mathbf{S}}}^{-1}(\mathbf{E}-\mathbf{b}) .
$$

We estimated the nine basic calibration parameters and the three Euler angles in bins of 30 days. For data equatorward of $\pm 55^{\circ}$ QD latitude, we performed a vector calibration using the component residuals of $\mathbf{B}_{\mathrm{RTP}}$ for estimating the model parameters (see "Model parameter estimation" section). In contrast, for data poleward of $\pm 55^{\circ}$ QD latitude, we performed a scalar calibration using the residuals of the vector magnitude, in which case the rotation matrices from the VFM to the RTP frame including the Euler angles disappear:

$$
\begin{aligned}
F & =\left|\mathbf{B}_{\mathrm{RTP}}\right|=\sqrt{\mathbf{B}_{\mathrm{RTP}}^{\mathrm{T}} \mathbf{B}_{\mathrm{RTP}}} \\
& =\sqrt{(\mathbf{E}-\mathbf{b})^{\mathrm{T}} \underline{\underline{\mathbf{S}}}^{-1}\left(\underline{\underline{\mathbf{P}}}^{-1}\right)^{\mathrm{T}} \underline{\underline{\mathbf{P}}}^{-1} \underline{\underline{\mathbf{S}}}^{-1}(\mathbf{E}-\mathbf{b})}
\end{aligned}
$$

at the expense of loosing the ability to estimate the Euler angles.

Table 1 summarizes the different parts of the model and the corresponding number of parameters.

\section{Model parameter estimation}

The geomagnetic field model parameters p, the Euler angles $\mathbf{q}$, and the calibration parameters $\mathbf{e}$ were derived by solving the least-squares problem: 
Table 1 Details on the parameterization of the individual model parts. Here, the number of basic parameters refers to the number of parameters irrespective of an explicit time-dependence

\begin{tabular}{|c|c|c|c|c|}
\hline \multicolumn{2}{|c|}{ Description of the model parameters } & \multirow{2}{*}{\begin{tabular}{|l|}
$\begin{array}{l}\text { Number of basic } \\
\text { parameters }\end{array}$ \\
255
\end{tabular}} & \multirow{2}{*}{$\begin{array}{l}\text { Temporal parameterization } \\
\text { order-6 B-spline }\end{array}$} & \multirow{2}{*}{$\begin{array}{l}\begin{array}{l}\text { Number } \\
\text { of parameters }\end{array} \\
6375\end{array}$} \\
\hline Internal field & Time-dependent $(n \leq 15)$ & & & \\
\hline & Static $(16 \leq n \leq 50)$ & 2345 & None & 2345 \\
\hline \multirow[t]{4}{*}{ External field } & SM degree-1 & 3 & RC-index & 3 \\
\hline & SM degree-2 & 5 & None & 5 \\
\hline & RC-baseline corrections & 3 & 80 bins (30 days) & 240 \\
\hline & GSM & 2 & None & 2 \\
\hline \multirow[t]{3}{*}{ Euler angles } & CHAMP & 3 & 33 bins (30 days) & 99 \\
\hline & Swarm-A & 3 & 50 bins (30 days) & 150 \\
\hline & Swarm-B & 3 & 50 bins (30 days) & 150 \\
\hline \multirow[t]{5}{*}{ Euler/Calibration } & CryoSat-2 FGM1 & 12 & 91 bins (30 days) & 1092 \\
\hline & CryoSat-2 FGM2 & 12 & 91 bins (30 days) & 1092 \\
\hline & CryoSat-2 FGM3 & 12 & 91 bins (30 days) & 1092 \\
\hline & GRACE-A & 12 & 120 bins (30 days) & 1440 \\
\hline & GRACE-B & 12 & 118 bins (30 days) & 1416 \\
\hline \multicolumn{4}{|c|}{ Total number of parameters (no platform magnetometer data) } & 9369 \\
\hline \multicolumn{4}{|c|}{ Total number of parameters } & 15501 \\
\hline
\end{tabular}

$$
\mathbf{m}^{*}=\underset{\mathbf{m}}{\operatorname{argmin}} \Phi(\mathbf{m}),
$$

where $\mathbf{m}=\left(\mathbf{p}^{\mathrm{T}}, \mathbf{q}^{\mathrm{T}}, \mathbf{e}^{\mathrm{T}}\right)^{\mathrm{T}}$ is the entire model parameter vector, and $\Phi$ is the cost function:

$$
\Phi(\mathbf{m})=(\mathbf{g}(\mathbf{p})-\mathbf{d}(\mathbf{q}, \mathbf{e}))^{\mathrm{T}}{\underline{\underline{\mathbf{C}_{\mathrm{d}}}}}^{-1}(\mathbf{g}(\mathbf{p})-\mathbf{d}(\mathbf{q}, \mathbf{e}))+\mathbf{m}^{\mathrm{T}} \underline{\underline{\underline{\Lambda}}},
$$

which penalizes a quadratic form in the residuals-the difference between the computed geomagnetic field model values $\mathbf{g}(\mathbf{p})$ and the calibrated, aligned magnetic data $\mathbf{d}(\mathbf{q}, \mathbf{e})$ - using the inverse of the data covariance matrix $\mathbf{C}_{\mathrm{d}}$, and a quadratic form in the model parameter vector using the regularization matrix $\Lambda$. For the definition of the matrices $\underline{C}_{\mathrm{d}}$ and $\underline{\underline{\Lambda}}$, see, respectively, "Data weighting" and "Model regularization" sections.

The least-squares solution $\mathbf{m}^{*}$ in Eq. (15) is found through an iterative quasi-Newton method, which consists of updating the model parameter vector $\mathbf{m}_{k}$ at iteration $k$ using $\mathbf{m}_{k+1}=\mathbf{m}_{k}+\Delta \mathbf{m}$ together with:

$$
\begin{aligned}
\Delta \mathbf{m}=( & \left.\left(\underline{\underline{\mathbf{G}}}_{k}\right)^{\mathrm{T}}{\underline{\underline{\mathbf{C}_{\mathrm{d}}}}}^{-1} \underline{\underline{\mathbf{G}}}_{k}+\underline{\underline{\Lambda}}\right)^{-1} \\
& \cdot\left(\left(\underline{\underline{\mathbf{G}_{k}}}\right)^{\mathrm{T}} \underline{\underline{\underline{\mathbf{C}_{\mathrm{d}}}}}{ }^{-1}\left(\mathbf{d}_{k}-\mathbf{g}_{k}\right)-\underline{\underline{\Lambda}} \mathbf{m}_{k}\right),
\end{aligned}
$$

where $\mathbf{d}_{k}=\mathbf{d}\left(\mathbf{q}_{k}, \mathbf{e}_{k}\right), \mathbf{g}_{k}=\mathbf{g}\left(\mathbf{p}_{k}\right)$, and $\underline{\underline{G}}_{k}$ is a matrix with entries corresponding to the partial derivative of the $i$ th residual with respect to the $j$ th model parameter:

$$
\left(\underline{\underline{G}}_{k}\right)_{i j}=\left.\frac{\partial(\mathbf{g}(\mathbf{p})-\mathbf{d}(\mathbf{q}, \mathbf{e}))_{i}}{\partial(\mathbf{m})_{j}}\right|_{\mathbf{m}=\mathbf{m}_{k}}
$$

evaluated at iteration $k$ (Tarantola 2005, p. 69). Some entries of $\underline{\mathbf{G}}_{k}$ are zero owing to data subsets that do not provide information on parts of the model. For example, scalar data do not constrain the Euler angles and vector data from one magnetometer do not constrain the Euler angles associated with another magnetometer. With the same idea in mind, we modified entries of $\underline{\underline{\mathbf{G}}}_{k}$ to prevent some data subsets from constraining certain parts of the internal field model. In particular, we set entries to zero for the following criteria:

1 The row index of the matrix entry corresponded to dayside data from a platform magnetometer, onboard CryoSat-2, or GRACE, and the column index corresponded to model parameters that describe the internal and external magnetic field. Therefore, the dayside data were only used to constrain the Euler angles and calibration parameters of the respective platform magnetometer.

2 The row index of the matrix entry corresponded to data from a platform magnetometer, on-board CryoSat-2 or GRACE, and the column index corresponded to the $\mathrm{B}$-spline parameters that param- 
Table 2 Overview of which data subset constrained which part of the model. The cross refers to non-zero entries in the matrix of partial derivatives, whereas the circle refers to zeros. The SV data refer to the annual difference of the revised monthly means

\begin{tabular}{|c|c|c|c|c|c|c|}
\hline \multicolumn{2}{|c|}{ Description of the model parameters } & \multicolumn{2}{|c|}{ Non-polar satellite data } & \multicolumn{2}{|c|}{ Polar satellite data } & \multirow[t]{2}{*}{ SV data } \\
\hline & & Day & Night & Day & Night & \\
\hline \multirow[t]{2}{*}{ Internal field } & Time-dependent $(n \leq 15)$ & $\bigcirc$ & $x^{1}$ & 0 & $x^{1}$ & $x$ \\
\hline & Static $(16 \leq n \leq 50)$ & $\bigcirc$ & $x$ & $\bigcirc$ & $x$ & $\bigcirc$ \\
\hline \multirow[t]{2}{*}{ External field } & SM & $\bigcirc$ & $x$ & $\bigcirc$ & $x$ & $\bigcirc$ \\
\hline & GSM & $\bigcirc$ & $x$ & $\bigcirc$ & $x$ & $\bigcirc$ \\
\hline \multirow[t]{8}{*}{ Euler angles } & CHAMP & $\bigcirc$ & $x$ & $\bigcirc$ & $\bigcirc$ & $\bigcirc$ \\
\hline & Swarm-A & $\bigcirc$ & $x$ & $\bigcirc$ & $\bigcirc$ & $\bigcirc$ \\
\hline & Swarm-B & $\bigcirc$ & $x$ & $\bigcirc$ & $\bigcirc$ & $\bigcirc$ \\
\hline & CryoSat-2 FGM1 & $x$ & $x$ & $\bigcirc$ & 0 & $\bigcirc$ \\
\hline & CryoSat-2 FGM2 & $x$ & $x$ & 0 & $\bigcirc$ & $\bigcirc$ \\
\hline & CryoSat-2 FGM3 & $x$ & $x$ & $\bigcirc$ & $\bigcirc$ & $\bigcirc$ \\
\hline & GRACE-A & $x$ & $x$ & 0 & $\bigcirc$ & 0 \\
\hline & GRACE-B & $x$ & $x$ & $\bigcirc$ & $\bigcirc$ & $\bigcirc$ \\
\hline \multirow[t]{5}{*}{ Calibration } & CryoSat-2 FGM1 & $x$ & $x$ & $x$ & $x$ & $\bigcirc$ \\
\hline & CryoSat-2 FGM2 & $x$ & $x$ & $x$ & $x$ & $\bigcirc$ \\
\hline & CryoSat-2 FGM3 & $x$ & $x$ & $x$ & $x$ & $\bigcirc$ \\
\hline & GRACE-A & $x$ & $x$ & $x$ & $x$ & 0 \\
\hline & GRACE-B & $x$ & $x$ & $x$ & $x$ & $\bigcirc$ \\
\hline
\end{tabular}

${ }^{1}$ Entries related to $g_{1}^{0}$ B-spline coefficients and platform magnetometer data are zero

eterize the $g_{1}^{0}$ Gauss coefficient of the internal field in time. Therefore, no platform magnetometer data were used to constrain the B-spline coefficients of the axial dipole which we believe are well determined using ground observatory data.

Table 2 gives an overview of whether or not certain datasets constrained specific parts of the model.

Nevertheless, we used the full model description in the forward evaluation to compute the residuals.

The iterative procedure described in Eq. (17) requires a starting model $\mathbf{m}_{0}$ to initialize the model parameter estimation. We initialized the internal field model parameters using the corresponding part of CHAOS-6-x9, while we set the external field model parameters to zero. To initialize the Euler angles, we used the values from CHAOS6-x9 in case of Swarm and CHAMP satellites, or set the angles to zero in case of CryoSat-2 and the GRACE satellite duo. For the calibration parameters, we simply set the offsets and non-orthogonalities to zero and the sensitivities to one over the whole time span. The parameter estimation usually converged after 10-15 iterations. We also tested other starting models, e.g., random calibration parameters, but found that our choice had little impact on the converged model parameters other than increasing the number of necessary iterations.
Table 3 Chosen values of $\sigma$ and $\psi$ for the different satellites. The values under Swarm apply to the data from the two Swarm satellites in this study (Swarm-A and Swarm-B), the values under CryoSat-2 to the data of the three magnetometers (FGM1, FGM2 and FGM3), and the values under GRACE to the data from both GRACE satellites (GRACE-A and GRACE-B)

\begin{tabular}{lllcc}
\hline & CHAMP & Swarm & CryoSat-2 & GRACE \\
\hline$\sigma(\mathrm{nT})$ & 2.5 & 2.2 & 6 & 10 \\
$\psi(\operatorname{arcsec})$ & 10 & 5 & 30 & 100 \\
\hline
\end{tabular}

\section{Data weighting}

For the vector components of the non-polar satellite data, we used a covariance matrix that accounts for the attitude uncertainty of the star cameras:

$$
\underline{\underline{\mathbf{C}_{\mathrm{B} 23}}}=\operatorname{diag}\left(\sigma^{2}, \sigma^{2}+B^{2} \psi^{2}, \sigma^{2}+B^{2} \psi^{2}\right)
$$

with respect to the $\mathrm{B} 23$ reference frame defined by unit vectors in the direction of $\mathbf{B}, \mathbf{n} \times \mathbf{B}$, and $\mathbf{n} \times(\mathbf{n} \times \mathbf{B})$, where $\mathbf{n}$ is an arbitrary unit vector not parallel to $\mathbf{B}$ that we chose to be the third CRF base vector, $\sigma^{2}$ is the variance of an isotropic instrument error and $\psi^{2}$ is the variance associated with random rotations around the three 
reference axes (Holme and Bloxham 1996). Table 3 summarizes the values of $\sigma$ and $\psi$ for the different satellite datasets.

We scaled the diagonal entries of the covariance matrix with Huber weights (Constable 1988; Sabaka et al. 2004) that we calculated for each component in the B23 reference frame to downweight data points that greatly deviated from the model evaluated at the previous iteration. After inverting and rotating the Huber-weighted covariance matrix of the individual data point into the RTP frame, we arranged them into a block-diagonal matrix completing the desired inverse data covariance matrix $\underline{\underline{\mathrm{C}}}^{-1}$. In case of the vector magnitude of the polar satellite data, we simply used $\sigma^{2}$ scaled with Huber weights as variance. The covariance of the ground observatory SV vector data was derived from detrended residuals to the CHAOS-6- $x 9$ model, including the covariance between vector components at a given location.

\section{Model regularization}

The regularization in the form of the matrix $\underline{\Lambda}$ in Eq. (15) is designed to ensure the convergence of the model parameter estimation by limiting the flexibility of the model. The regularization matrix is block diagonal and consists of the blocks $\Lambda_{\text {int }}, \underline{\underline{\text { ext }}}$, and $\Lambda_{\text {cal }}$, which regularized the internal, external, and the calibration parameters, respectively. We did not regularize the Euler angles, such that corresponding blocks in the regularization matrix are zero.

Turning to the internal part of the model, following the example of earlier models in the CHAOS series, we designed a regularization based on the square of the third time-derivative of the radial field component $B_{r}$ integrated over the core mantle boundary (CMB) and averaged over the entire model time span:

$$
\left\langle\dddot{B}_{r}^{2}\right\rangle=\frac{1}{4 \pi\left(t_{\mathrm{e}}-t_{\mathrm{s}}\right)} \int_{t_{\mathrm{s}}}^{t_{\mathrm{e}}} \int_{\Omega(c)}\left(\frac{\partial^{3} B_{r}}{\partial t^{3}}\right)^{2} \mathrm{~d} \Omega \mathrm{d} t,
$$

where $c=3485.0 \mathrm{~km}$ is the chosen spherical reference radius of the CMB, $\Omega(c)$ denotes the CMB given as the spherical surface of radius $c$, and $\mathrm{d} \Omega=\sin \theta \mathrm{d} \theta \mathrm{d} \phi$ is the surface element for the integration. Furthermore, we set up a regularization of the internal field based on the square of the second time-derivative of the radial component integrated over the CMB at the model start time $t_{\mathrm{s}}$ :

$$
\left\langle\ddot{B}_{r}^{2}\left(t_{\mathrm{s}}\right)\right\rangle=\frac{1}{4 \pi} \int_{\Omega(c)}\left(\left.\frac{\partial^{2} B_{r}}{\partial t^{2}}\right|_{t=t_{\mathrm{s}}}\right)^{2} \mathrm{~d} \Omega,
$$

and similarly for the end time by replacing $t_{\mathrm{s}}$ with $t_{\mathrm{e}}$. Returning to Eq. (20), thanks to the orthogonality of spherical harmonics on the surface of the sphere, carrying out the spatial integration leads to:

$$
\left\langle\dddot{B}_{r}^{2}\right\rangle=\sum_{n=1}^{N_{\text {int }}}\left(w_{\Omega}(n) \sum_{m=0}^{n}\left(\left\langle\dddot{g}_{n}^{m}(t)^{2}\right\rangle_{t}+\left\langle\dddot{h}_{n}^{m}(t)^{2}\right\rangle_{t}\right)\right),
$$

where $w_{\Omega}=\frac{(n+1)^{2}}{2 n+1}\left(\frac{a}{c}\right)^{2 n+4}$ is a spatial factor that follows from the surface integration and $\langle\cdot\rangle_{t}=\frac{1}{t_{\mathrm{e}}-t_{\mathrm{s}}} \int_{t_{\mathrm{s}}}^{t_{\mathrm{e}}} \mathrm{d} t$ denotes the time average. Utilizing the fact that the timedependence of the Gauss coefficients is given by sixthorder B-splines, terms such as:

$$
\begin{aligned}
\left\langle\dddot{g}_{n}^{m}(t)^{2}\right\rangle_{t} & =\sum_{j, j^{\prime}} g_{n, j}^{m} g_{n, j^{\prime}}^{m}\left(\dddot{B}_{6, j}(t) \dddot{B}_{6, j^{\prime}}(t)\right\rangle_{t} \\
& =\sum_{j, j^{\prime}} g_{n, j}^{m} g_{n, j^{\prime}}^{m} A_{j j^{\prime}} \\
& =\left(\mathbf{g}_{n}^{m}\right)^{\mathrm{T}} \underline{\underline{\mathbf{A}_{t}}} \mathbf{g}_{n}^{m}
\end{aligned}
$$

can be written as a quadratic form in $\mathbf{g}_{n}^{m}=\left(g_{n, 1}^{m}, g_{n, 2}^{m}, \ldots\right)^{\mathrm{T}}$, the vector of the spline coefficients of $g_{n}^{m}$, using the matrix $\underline{\underline{\mathbf{A}_{t}}}$ that has entries corresponding to the time averages of products of the third time-derivative of the B-splines. While the time-derivatives of the B-splines are known analytically, we approximated the time average numerically by a Riemann sum of rectangles. A similar computation of Eq. (21), now evaluating the derivatives only at the endpoints instead of averaging in time, yields matrices $\left(\underline{\underline{\mathbf{A}_{t_{\mathrm{s}}}}}\right)_{j j^{\prime}}=\ddot{B}_{6, j}\left(t_{\mathrm{s}}\right) \ddot{B}_{6, j^{\prime}}\left(t_{\mathrm{s}}\right)$ and $\left(\underline{\underline{\mathbf{A}_{t_{\mathrm{e}}}}}\right)_{j j^{\prime}}=\ddot{B}_{6, j}\left(t_{\mathrm{e}}\right) \ddot{B}_{6, j^{\prime}}\left(t_{\mathrm{e}}\right)$. Finally, based on the physical quantities in Eqs. (20) and (21), we devised a block-diagonal regularization matrix for the internal magnetic field model:

$$
\begin{aligned}
& \underline{\underline{\Lambda_{\mathrm{int}}}}=\operatorname{diag}( w_{\Omega}(n) w_{m}(m) w_{\mathrm{tp}}(n) \\
&\left.\cdot\left(\lambda_{t} \underline{\underline{\mathbf{A}_{t}}}+\lambda_{t_{\mathrm{s}}} \underline{\underline{\mathbf{A}_{t_{\mathrm{s}}}}}+\lambda_{t_{\mathrm{e}}} \underline{\underline{\mathbf{A}_{t_{\mathrm{e}}}}}\right)\right),
\end{aligned}
$$

where $n$ and $m$ run over the degree and order in the spherical harmonic expansion of the internal field in Eq. (1); $w_{m}(m)$ and $w_{\text {tp }}(n)$ are functions which control the regularization strength based on the degree and order of the internal Gauss coefficients; $\lambda_{t}, \lambda_{t_{\mathrm{s}}}$, and $\lambda_{t_{\mathrm{e}}}$ are parameters that, respectively, set the regularization strength over the entire model time span, at the model start time and end time. Following Finlay et al. (2020), to relax the regularization at higher spherical harmonic degree, we defined $w_{\mathrm{tp}}(n)$ as a tapered window which gradually reduces from one to 0.005 : 


$$
w_{\mathrm{tp}}(n)=\left\{\begin{array}{l}
1, \quad n<n_{\min } \\
\tau(n), \quad n_{\min } \leq n \leq n_{\max } \\
0.005, \quad n>n_{\max },
\end{array}\right.
$$

where $n_{\min }=3$ and $n_{\max }=6$ are the chosen limits of a half-cosine taper:

$$
\tau(n)=\frac{0.995}{2}\left[1+\cos \left(\pi \frac{n-n_{\min }}{n_{\max }-n_{\min }}\right)\right]+0.005 .
$$

In contrast to Finlay et al. (2020), who used $n_{\max }=11$ to achieve stable power spectra with more power in the time-dependence of the high-degree coefficients without causing instabilities, we were able to further decrease the upper limit of the taper. The magnetospheric and ionospheric field and their induced counterparts may also cause the estimation of the internal field parameters to become unstable. Our experience shows that it is typically the zonal harmonics that become unstable first if the regularization is not sufficiently strong. Therefore, in addition to the degree-dependent temporal regularization, there is a special treatment of zonal and non-zonal spherical harmonics based on:

$$
w_{m}(m)= \begin{cases}\lambda_{0}, & m=0 \\ \lambda_{m}, & m \neq 0 .\end{cases}
$$

Note that the regularization of the internal field model only constrains the time-derivatives of the field but not the field itself.

Turning to the external part of the model, we regularized only the bin-to-bin variability of the three RCbaseline corrections $\Delta q_{1, \mathrm{SM}}^{0}, \Delta q_{1, \mathrm{SM}}^{1}$, and $\Delta s_{1, \mathrm{SM}}^{1}$ in Eq. (3) using a quadratic form in the first forward difference of neighboring bins. The forward difference was calculated with the matrix:

$$
\underline{\underline{\mathbf{D}}}=\frac{1}{t_{\mathrm{e}}-t_{\mathrm{s}}}\left(\begin{array}{cccc}
-1 & 1 & & \\
& \ddots & \ddots & \\
& & -1 & 1
\end{array}\right),
$$

whose number of columns is equal to the number of bins that comprise each RC-baseline correction. Taken together, the regularization matrix for all parameters related to the external field model reads:

$$
\underline{\underline{\Lambda_{\text {ext }}}}=\operatorname{diag}\left(0, \ldots, 0, \lambda_{\text {ext }} \underline{\underline{\mathbf{I}_{3}}} \otimes \underline{\underline{\mathbf{D}_{2}}}, 0, \ldots, 0\right),
$$

where $\otimes$ is the Kronecker product, $\underline{\mathbf{I}}_{3}$ is the unit matrix of size three corresponding to the three $\mathrm{RC}$-baseline corrections, $\underline{\mathbf{D}}_{2}=\underline{\underline{\mathbf{D}}}^{\mathrm{T}} \underline{\underline{\mathbf{D}}}$ is the coefficient matrix that determines the quadratic form, additional zeros on the diagonal indicate the other unregularized model parameters of the external field, and $\lambda_{\text {ext }}$ is the chosen regularization parameter.

Turning to the calibration parameters, we regularized a quadratic form in the bin-to-bin variability of each calibration parameter for the five platform magnetometers (three on CryoSat-2 and one on each of the two GRACE satellites). The regularization matrix $\Lambda_{\text {cal }}$ is block-diagonal with each block $\underline{\underline{\Lambda_{\mathrm{cal}, i}}}, i=1, \ldots, \overline{\overline{5, \text { cor- }}}$ responding to the calibration parameters for each of the five platform magnetometers. The regularization matrix can be written as:

$$
\begin{aligned}
& \underline{\underline{\Lambda_{\mathrm{cal}}}}=\operatorname{diag}\left(\underline{\underline{\Lambda_{\mathrm{cal}, 1}}}, \ldots, \underline{\underline{\Lambda_{\mathrm{cal}, 5}}}\right) \\
& \underline{\underline{\Lambda_{\text {cal }, i}}}=\operatorname{diag}\left(\lambda_{b, i}, \lambda_{s, i}, \lambda_{u, i}\right) \otimes \underline{\underline{\mathbf{I}_{3}}} \otimes \underline{\underline{\mathbf{D}_{2}}},
\end{aligned}
$$

where we define the regularization parameters $\lambda_{b, i}, \lambda_{s, i}$ and $\lambda_{u, i}$ to control the temporal smoothness of the offsets, sensitivities, and non-orthogonalities, respectively.

\section{Results and discussion}

We built two geomagnetic field models which span 10 years from the 1st of January 2008 to the 31st of December 2018, but differ in the use of platform magnetometer data to constrain the field model parameters.

The first model, Model-A, was derived with data from the Swarm-A, Swarm-B, and CHAMP satellites, and the monthly SV data from ground observatories. It served as a reference model, which allowed us to identify differences to models which were derived using platform magnetometer data in addition. Considering the model parameterization, regularization, and estimation, ModelA is very similar to the CHAOS model series. In fact, the parameterization of the geomagnetic field and the alignment parameters of the satellite data are identical, except for the lower truncation degree of the internal field and the longer bins of the alignment parameters and $\mathrm{RC}$-baseline corrections in Model-A. A notable difference is the use of gradient data in the CHAOS model. The strong temporal regularization of the high-degree Gauss coefficients of the time-dependent internal field has been relaxed in the newly released CHAOS-7 model through a taper, which we also used here. For Model-A, we tuned the regularization, such that the model parameters matched the ones of the CHAOS-6- $x 9$ model as close as possible. Table 4 shows the numerical values of the regularization parameters.

The second model, Model- $B$, is our preferred model and was derived with data from Swarm-A, Swarm-B, CHAMP, monthly ground observatory SV data, and, as opposed to Model-A, platform magnetometer data from CryoSat-2 FGM1, CryoSat-2 FGM2, CryoSat-2 FGM3, GRACE-A, and GRACE-B. In addition to Model-A and 
Table 4 Chosen numerical values of the regularization parameters. The values are valid for all the models built in this paper insofar as the regularization terms are applicable to the specific model

\begin{tabular}{|c|c|c|}
\hline \multicolumn{2}{|c|}{ Description of the model parameters } & \multirow{2}{*}{$\begin{array}{l}\text { Regularization parameter } \\
\lambda_{t}=1.0\left(\frac{\mathrm{n} T}{\mathrm{y} \mathrm{r}^{3}}\right)^{-2}, \lambda_{t_{\mathrm{s}}}=0.03\left(\frac{\mathrm{nT}}{\mathrm{y} \mathrm{r}^{2}}\right)^{-2}, \lambda_{t_{\mathrm{e}}}=0.03\left(\frac{\mathrm{nT}}{\mathrm{y} \mathrm{r}^{2}}\right)^{-2} \\
\lambda_{0}=60, \lambda_{\mathrm{m}}=0.65\end{array}$} \\
\hline Internal field & Time-dependent & \\
\hline External field & RC-baseline corrections & $\lambda_{\mathrm{ext}}=4 \times 10^{5}\left(\frac{\mathrm{nT}}{\mathrm{yr}}\right)^{-2}$ \\
\hline \multirow[t]{5}{*}{ Calibration $^{1}$} & CryoSat-2 FGM1 & $\lambda_{b}=9.1 \times 10^{2}\left(\frac{\mathrm{eu}}{\mathrm{yr}}\right)^{-2} \lambda_{s}=9.1 \times 10^{10}\left(\frac{\mathrm{eu}}{\mathrm{nTyr}}\right)^{-2} \lambda_{u}=2.8 \times 10^{2}\left(\frac{1^{\circ}}{\mathrm{yr}}\right)^{-2}$ \\
\hline & CryoSat-2 FGM2 & $\lambda_{b}=9.1 \times 10^{2}\left(\frac{\mathrm{eu}}{\mathrm{yr}}\right)^{-2} \lambda_{s}=9.1 \times 10^{10}\left(\frac{\mathrm{eu}}{\mathrm{nTyr}}\right)^{-2} \lambda_{u}=2.8 \times 10^{2}\left(\frac{1^{\circ}}{\mathrm{yr}}\right)^{-2}$ \\
\hline & CryoSat-2 FGM3 & $\lambda_{b}=9.1 \times 10^{2}\left(\frac{\mathrm{eu}}{\mathrm{yr}}\right)^{-2} \lambda_{s}=9.1 \times 10^{10}\left(\frac{\mathrm{eu}}{\mathrm{nTyr}}\right)^{-2} \lambda_{u}=2.8 \times 10^{2}\left(\frac{1^{\circ}}{\mathrm{yr}}\right)^{-2}$ \\
\hline & GRACE-A & $\lambda_{b}=1.2 \times 10^{3}\left(\frac{\mathrm{eu}}{\mathrm{yr}}\right)^{-2}, \lambda_{s}=1.2 \times 10^{13}\left(\frac{\mathrm{eu}}{\mathrm{nTyr}}\right)^{-2}, \lambda_{u}=3.7 \times 10^{8}\left(\frac{1^{\circ}}{\mathrm{yr}}\right)^{-2}$ \\
\hline & GRACE-B & $\lambda_{b}=1.2 \times 10^{3}\left(\frac{\mathrm{eu}}{\mathrm{yr}}\right)^{-2} \lambda_{s}=1.2 \times 10^{13}\left(\frac{\mathrm{eu}}{\mathrm{nTyr}}\right)^{-2} \lambda_{u}=3.6 \times 10^{8}\left(\frac{1^{\circ}}{\mathrm{yr}}\right)^{-2}$ \\
\hline
\end{tabular}

${ }^{1}$ Not applicable to Model-A, which was not derived from platform magnetometer data
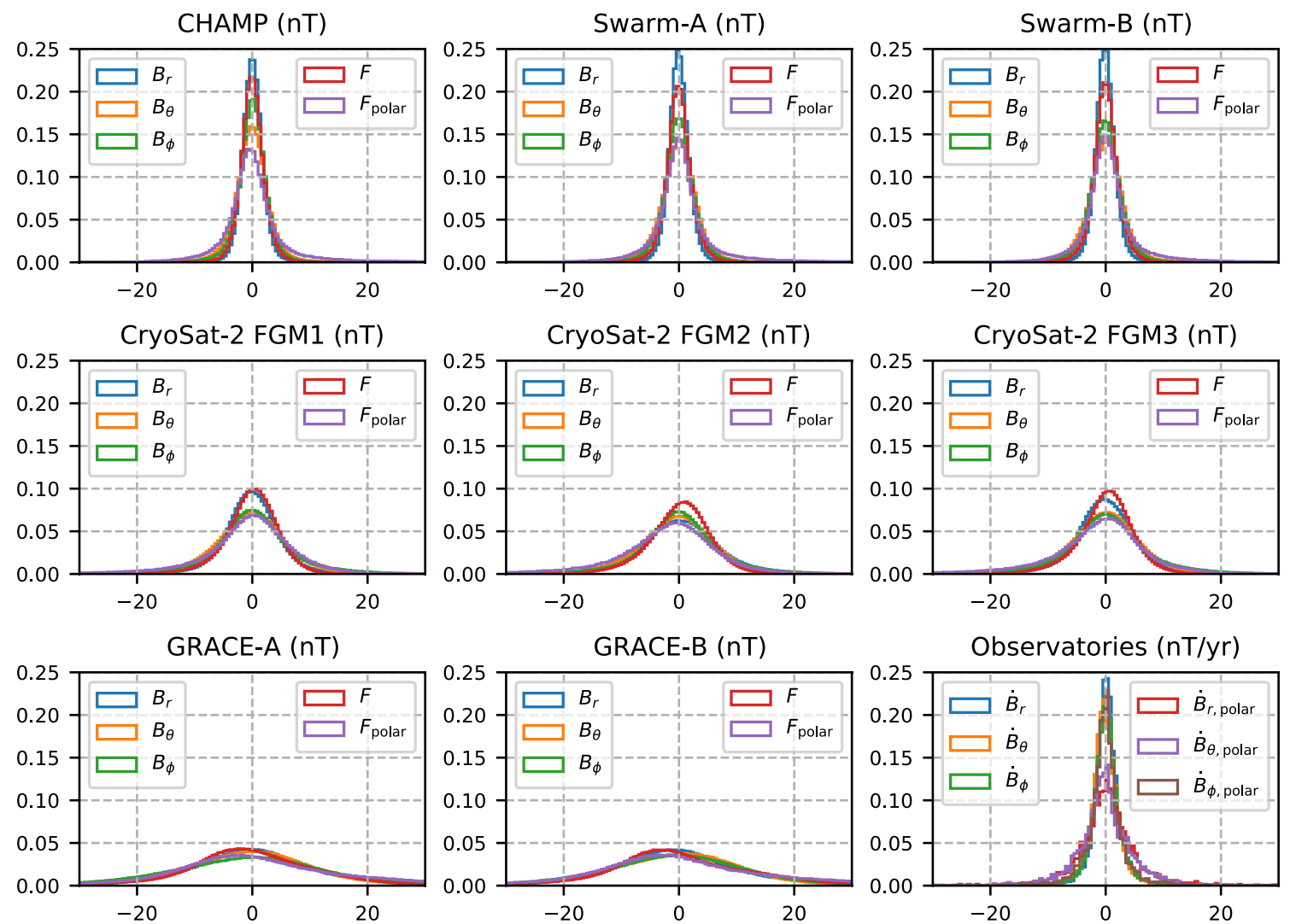

Fig. 3 Histograms of the residuals of each satellite and ground observatory SV data using Model-B. The histograms have been normalized to have unit area. Computed statistics are shown in Table 5 for the satellite data and Table 6 for the ground observatory SV data

Model-B, we built test models in a series of experiments to investigate the effect of platform magnetometer data on the estimation of the geomagnetic field model. Details of the test models are given below. The regularization parameters are the same for all the presented models, i.e., Model-A, Model-B, and the test models. 
Fit to satellite data and ground observatory SV data

We begin with reporting on the fit of Model-B to the satellite data and ground observatory SV data. The histograms of the scalar and vector residuals for each dataset are shown in Fig. 3.
The residuals of Swarm-A, Swarm-B, CHAMP, and the ground observatories show narrow and near-zero centered peaks, which demonstrate the high-quality and low-noise level of these datasets. In contrast, the peaks are broader for CryoSat- 2 and even more in the case of

Table 5 Number $\mathbf{N}$, Huber-weighted mean, and standard deviation $\sigma$ computed from the residuals of the satellite data for each vector component and split into polar (poleward $\pm 55^{\circ}$ ) and non-polar (equatorward $\pm 55^{\circ}$ ) $Q D$ latitudes. $N o t e$ that non-polar scalar data were not used in the model parameter estimation-statistics are only shown for completeness

\begin{tabular}{|c|c|c|c|c|c|}
\hline Dataset & Quasi-dipole latitude & Component & $\mathbf{N}$ & mean $(\mathrm{nT})$ & $\sigma(\mathrm{nT})$ \\
\hline \multirow[t]{5}{*}{ CHAMP } & Non-polar & $B_{r}$ & 707131 & 0.02 & 1.93 \\
\hline & & $B_{\theta}$ & 707131 & -0.11 & 2.84 \\
\hline & & $B_{\phi}$ & 707131 & 0.03 & 2.32 \\
\hline & & $F$ & 707131 & 0.01 & 1.93 \\
\hline & Polar & $F$ & 200084 & -0.02 & 5.10 \\
\hline \multirow[t]{5}{*}{ CryoSat-2 FGM1 } & Non-polar & $B_{r}$ & 958362 & -0.06 & 4.39 \\
\hline & & $B_{\theta}$ & 958362 & -0.31 & 5.76 \\
\hline & & $B_{\phi}$ & 958362 & 0.06 & 6.49 \\
\hline & & $F$ & 958362 & 0.06 & 4.18 \\
\hline & Polar & $F$ & 331097 & -0.28 & 7.56 \\
\hline \multirow[t]{5}{*}{ CryoSat-2 FGM2 } & Non-polar & $B_{r}$ & 958362 & -0.03 & 6.42 \\
\hline & & $B_{\theta}$ & 958362 & -0.29 & 6.01 \\
\hline & & $B_{\phi}$ & 958362 & 0.07 & 6.55 \\
\hline & & $F$ & 958362 & 0.18 & 4.86 \\
\hline & Polar & $F$ & 331097 & -1.70 & 8.21 \\
\hline \multirow[t]{5}{*}{ CryoSat-2 FGM3 } & Non-polar & $B_{r}$ & 958362 & -0.07 & 4.76 \\
\hline & & $B_{\theta}$ & 958362 & -0.23 & 5.71 \\
\hline & & $B_{\phi}$ & 958362 & 0.04 & 6.80 \\
\hline & & $F$ & 958362 & 0.12 & 4.35 \\
\hline & Polar & $F$ & 331097 & -1.01 & 7.86 \\
\hline \multirow[t]{5}{*}{ GRACE-A } & Non-polar & $B_{r}$ & 1082071 & -0.12 & 11.40 \\
\hline & & $B_{\theta}$ & 1082071 & -0.24 & 10.48 \\
\hline & & $B_{\phi}$ & 1082071 & -0.79 & 13.57 \\
\hline & & $F$ & 1082071 & -0.16 & 10.59 \\
\hline & Polar & $F$ & 356988 & 0.32 & 15.56 \\
\hline \multirow[t]{5}{*}{ GRACE-B } & Non-polar & $B_{r}$ & 997802 & -0.30 & 11.77 \\
\hline & & $B_{\theta}$ & 997802 & -0.69 & 11.09 \\
\hline & & $B_{\phi}$ & 997802 & -0.68 & 12.35 \\
\hline & & $F$ & 997802 & 0.02 & 11.53 \\
\hline & Polar & $F$ & 331516 & -0.24 & 15.56 \\
\hline \multirow[t]{5}{*}{ Swarm-A } & Non-polar & $B_{r}$ & 817400 & -0.03 & 1.65 \\
\hline & & $B_{\theta}$ & 817400 & -0.06 & 2.97 \\
\hline & & $B_{\phi}$ & 817400 & -0.02 & 2.59 \\
\hline & & $F$ & 817400 & -0.03 & 2.06 \\
\hline & Polar & $F$ & 218776 & 0.22 & 4.66 \\
\hline \multirow[t]{5}{*}{ Swarm-B } & Non-polar & $B_{r}$ & 809720 & -0.09 & 1.63 \\
\hline & & $B_{\theta}$ & 809720 & -0.05 & 3.02 \\
\hline & & $B_{\phi}$ & 809720 & -0.04 & 2.61 \\
\hline & & $F$ & 809720 & -0.01 & 2.03 \\
\hline & Polar & $F$ & 218106 & 0.30 & 4.29 \\
\hline
\end{tabular}


Table 6 Number $\mathbf{N}$, Huber-weighted mean, and standard deviation $\sigma$ computed from the residuals of the monthly ground observatory SV data for each component and split into polar (poleward $\pm 55^{\circ}$ ) and non-polar (equatorward $\pm 55^{\circ}$ ) $Q D$ latitudes

\begin{tabular}{llllcr}
\hline Dataset & Quasi-dipole latitude & Component & N & Mean (nT/yr) & $\boldsymbol{\sigma}(\mathbf{n T} / \mathbf{y r})$ \\
\hline Observatories & Non-polar & $\dot{B}_{r}$ & 11348 & 0.20 & 2.09 \\
& & $\dot{B}_{\theta}$ & 11348 & -0.18 & 0.06 \\
& & $\dot{B}_{\phi}$ & 11348 & 0.22 & 2.43 \\
& Polar & $\dot{B}_{r}$ & 3609 & -0.19 & 4.43 \\
& & $\dot{B}_{\theta}$ & 3609 & -0.08 & 2.85 \\
\hline
\end{tabular}

GRACE, which is, as expected, due to the higher data noise level. By separating the residuals poleward of $\pm 55^{\circ}$ QD latitude from the ones equatorward, we find that peaks are broader at polar QD latitudes for all datasets, which is a result of unmodeled magnetic signal of the polar ionospheric current system. Also, the histograms of the GRACE residuals are biased toward negative values. Upon further investigation, we found a local time-dependence especially visible in the scalar residuals, which could indicate that signals from solar array and battery currents have not been fully removed from the GRACE datasets used here. The residual statistics are summarized in Table 5 for the satellite data and Table 6 for the ground observatory SV data.

Figure 4 shows the time-series of the SV components at six chosen ground observatories together with the computed values from Model-A and Model-B.

Overall, the fit of Model-A and Model-B to the ground observatory SV data is good, as expected, for the first five observatory SV shown, since these data were used in the model parameter estimation. The computed values of Model-A and Model-B differ especially during the gap from 2010 to 2014, where Model-B can make use of platform magnetometer data in addition to the ground observatory SV data, while Model-A only relies on the ground observatories. That shows that platform magnetometer data contribute to the internal field model especially when there is a lack of calibrated satellite data from CHAMP and Swarm. Perhaps even more convincing is the performance of both models when compared to a dataset not used in the inversion. With the SV data from Saint Helena, we show such an independent dataset in the last row of Fig. 4. Although both models fit Saint Helena well, Model-B performs slightly better in the radial SV in 2013 and the azimuthal SV at least in the first half of the gap period, until 2012.

To summarize, with Model-B, we built a model that fits both the satellite and ground observatory SV data to a satisfactory level, which shows that platform magnetometer data can be successfully used in geomagnetic field modeling.

\section{Calibration parameters}

We document the estimated calibration parameters of each platform magnetometer dataset by showing the time-series in Fig. 5 and the respective mean values in Table 7.

In Fig. 5, the rows of panels correspond to the CryoSat-2 (top three) and GRACE (bottom two) platform magnetometer datasets, and the columns of panels show the offsets (left), sensitivities (middle), and non-orthogonality angles (right). Since Alken et al. (2020) also used magnetic data from the three platform magnetometers on-board CryoSat-2, it is possible to compare the estimated calibration parameters. First, comparing the timeaveraged values of the calibration parameters (Table 7 here and Table 4 in Alken et al. (2020)), we find that the non-orthogonalities are equal to within $0.01^{\circ}$ and the offsets to within $1 \mathrm{eu}$. The averaged values of sensitivities are equal to within $1 \times 10^{-4} \mathrm{eu} / \mathrm{nT}$ (notice that Alken et al. (2020) use the reciprocal of the sensitivity). In terms of the temporal variability, we find that our estimated calibration parameters have amplitudes that are smaller, or equal in case of the offsets, which is likely due to a difference in the regularization strength. In Fig. 5, we also show the CryoSat-2 calibration parameters of Olsen et al. (2020) for comparison. Again, the calibration parameters are very similar and differ only in the time variations (e.g., $s_{1}$ ) due to the choice of the regularization parameters of this study and Olsen et al. (2020). Given the acceptable fit to the platform magnetometer data and the reasonable temporal variability of the calibration parameters, we conclude that the calibration of the CryoSat-2 and GRACE platform magnetometers was successful.

\section{Results of the experiments}

We conducted a series of experiments in which we changed the model estimation, parameterization, and 


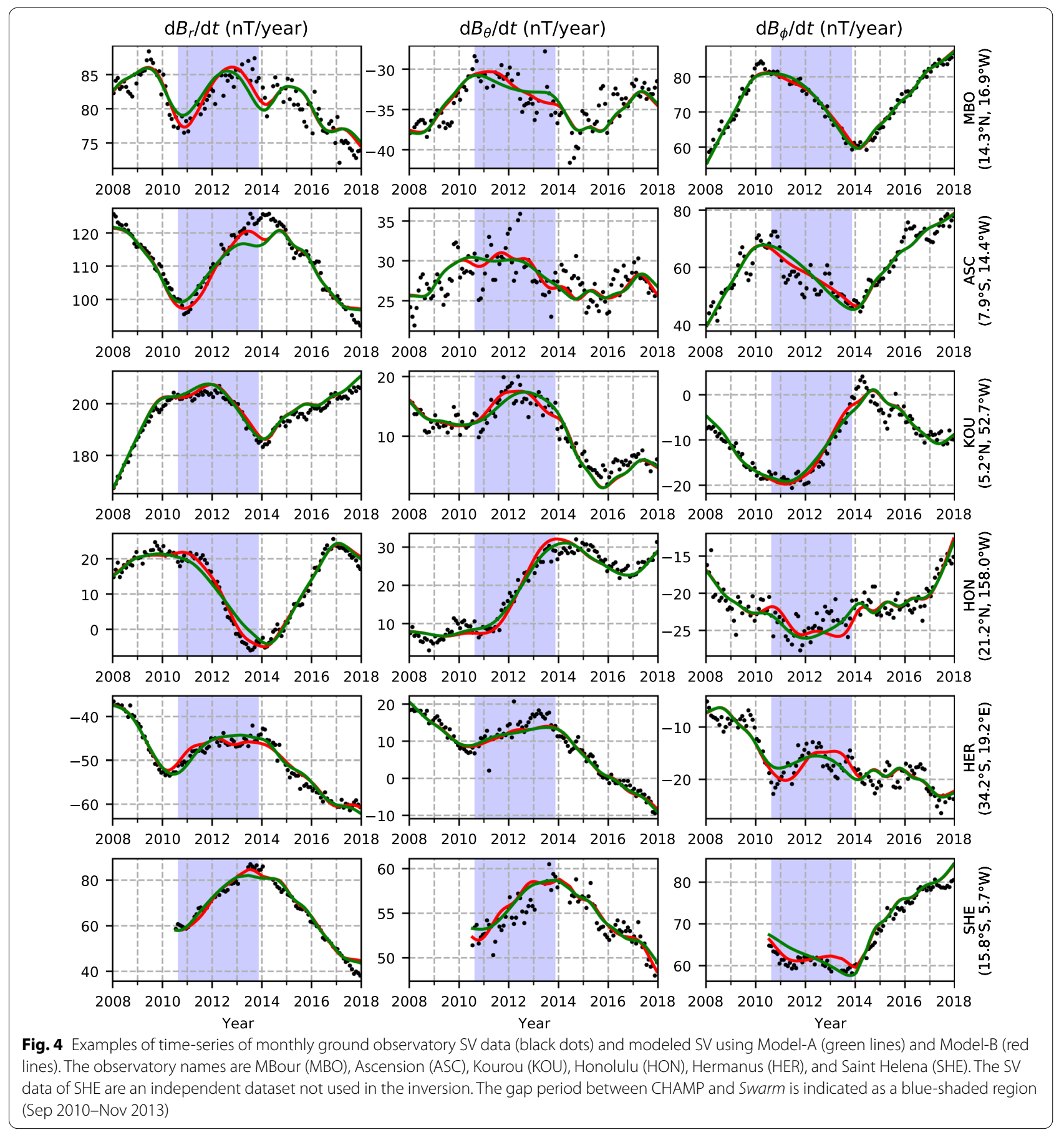

data selection with the goal to investigate and document difficulties when dealing with platform magnetometer data in a co-estimation scheme. This section also justifies the modeling strategies that went into the construction of our preferred geomagnetic field model, Model-B.

In a first experiment, we allowed the nightside platform magnetometer data to participate in the estimation of the axial dipole coefficient of the time-dependent internal field. That is, we derived a test model, Model-C, identical to Model-B but left the matrix of partial derivatives $\mathbf{G}$ unchanged, so that the entries corresponding to the B-spline coefficients $g_{1, j}^{0}$ were non-zero, and thus, the satellite data contributed to the estimation of the internal dipole coefficients. On the left of Fig. 6, we show the time-derivative of $g_{1}^{0}$ as a function of time computed with 


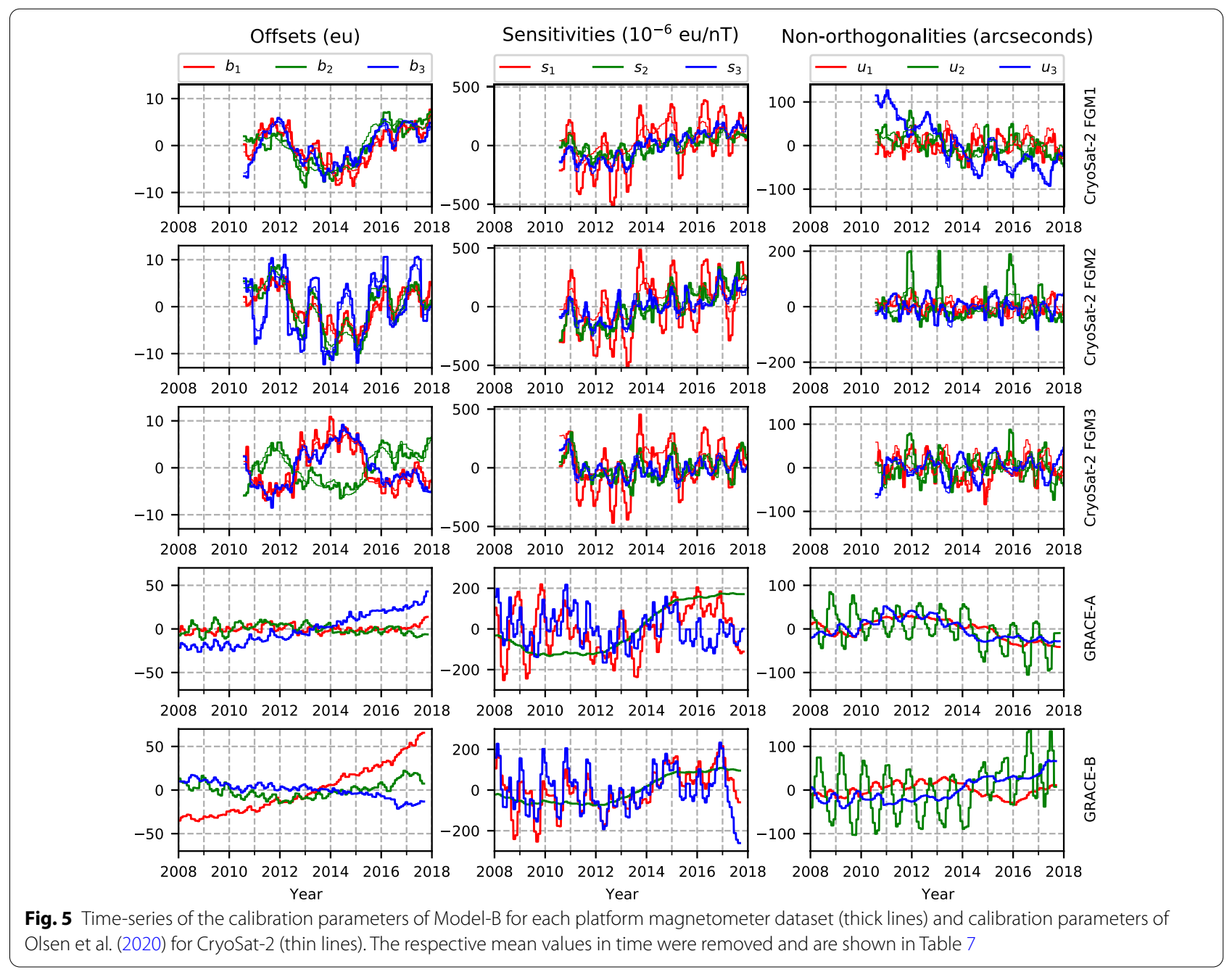

Table 7 Mean values of the calibration parameters for each platform magnetometer dataset. The time-series are shown in Fig. 5

\begin{tabular}{|c|c|c|c|c|c|c|c|c|c|}
\hline Dataset & $\begin{array}{l}b_{1} \\
(\mathrm{eu})\end{array}$ & $\begin{array}{l}b_{2} \\
(\mathrm{eu})\end{array}$ & $\begin{array}{l}b_{3} \\
(\mathrm{eu})\end{array}$ & $\begin{array}{l}s_{1} \\
(\mathrm{eu} / \mathrm{nT})\end{array}$ & $\begin{array}{l}s_{2} \\
(\mathrm{eu} / \mathrm{nT})\end{array}$ & $\begin{array}{l}s_{3} \\
(\mathrm{eu} / \mathrm{nT})\end{array}$ & $\begin{array}{l}u_{1} \\
\left({ }^{\circ}\right)\end{array}$ & $\begin{array}{l}u_{2} \\
\left({ }^{\circ}\right)\end{array}$ & $\begin{array}{l}u_{3} \\
\left({ }^{\circ}\right)\end{array}$ \\
\hline CryoSat-2 FGM1 & 5.0 & 165.6 & -10.7 & 1.005178 & 1.004851 & 1.004479 & 0.453 & 0.191 & -0.336 \\
\hline CryoSat-2 FGM2 & 77.6 & -16.6 & 61.8 & 1.004697 & 1.003993 & 1.003427 & -0.288 & 0.050 & 0.502 \\
\hline CryoSat-2 FGM3 & -115.2 & -29.4 & -44.6 & 1.000863 & 1.005424 & 1.002168 & 0.745 & -0.045 & -0.000 \\
\hline GRACE-A & 746.4 & -2632.1 & -2310.0 & 1.034238 & 1.032041 & 1.018168 & -0.251 & -0.161 & 0.048 \\
\hline GRACE-B & 406.0 & -2622.0 & -2005.6 & 1.029785 & 1.026781 & 1.017845 & -0.056 & -0.209 & 0.106 \\
\hline
\end{tabular}

Model-B and Model-C, while, on the right, we show $s_{1}$ of GRACE-A as an example of the calibration parameters.

In contrast to Model-B, Model- $C$ features a conspicuous detour of the time-derivative of the $g_{1}^{0}$ coefficient in the gap between CHAMP and Swarm data (blue-shaded region). Although we only show $s_{1}$ of GRACE-A in Fig. 6, we find that all three sensitivities of each platform magnetometer differ in the gap period between Model-C and Model-B. The other internal Gauss coefficients also deviate but to a lesser extent. Interestingly, other model parameters such as the offsets, non-orthogonality angles, Euler angles, and external field parameters seem 

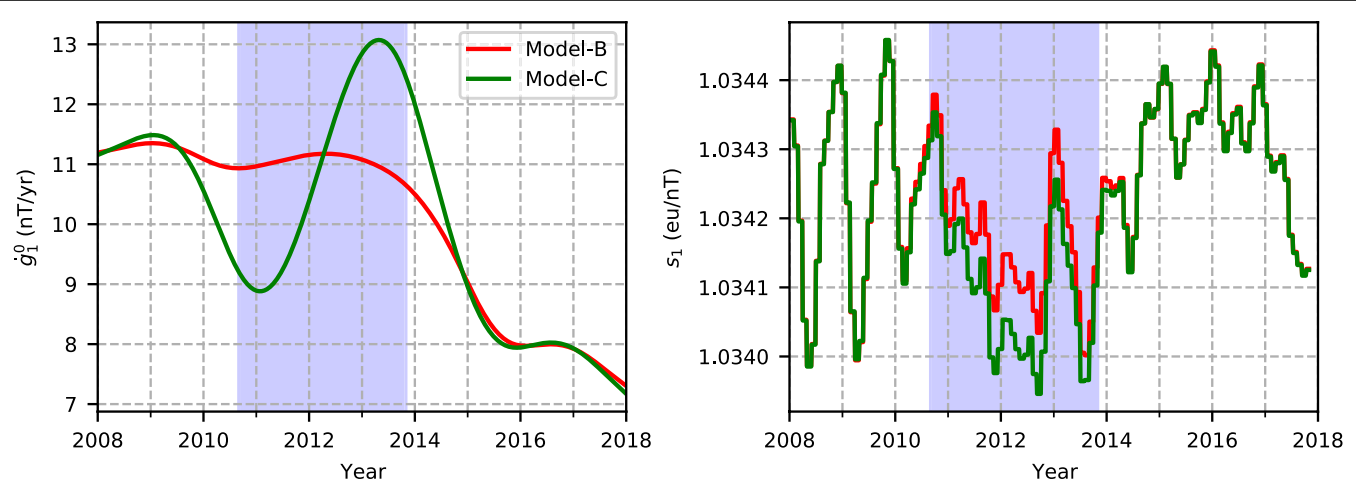

Fig. 6 Time-derivative of $g_{1}^{0}$ (left) and sensitivity $s_{1}$ for GRACE-A as computed from Model-B and Model-C (right). For Model-C, we allowed nightside platform magnetometer data to contribute to the estimation of the internal $g_{1}^{0}$ Gauss coefficient. The gap period between CHAMP and Swarm is indicated as a blue-shaded region (Sep 2010 to Nov 2013)

qualitatively unaffected. The same correlation between the internal axial dipole coefficient and the sensitivities has been reported by Alken et al. (2020) who show that this effect can be mitigated either by including large amounts of previously calibrated data or through the use of a regularization that favors a linear time-dependence of the internal dipole during the gap period. Due to the lack of additional calibrated data and our interest in the high-degree SA during the gap that such a regularization affects by redistributing power to higher degrees, we chose to set the dependence of $g_{1}^{0}$, the most affected internal Gauss coefficient, on the satellite platform magnetometer data to zero. In other words, we completely relied on the ground observatory SV data and the temporal regularization to estimate the time-dependence of $g_{1}^{0}$ in the gap period.

In a second experiment, we built a test model, Model$\mathrm{D}$, which uses 30 day bins of the RC-baseline corrections consistently over the whole model time span in contrast to Model-A and Model-B, which use a single bin spanning the entire gap period. As an example, Fig. 7 shows the RC-baseline correction $\Delta q_{1}^{0}$ on the left and the calibration parameter $s_{1}$ of GRACE-A on the right, computed with Model-D and Model-B.

In Model-D, $\Delta q_{1}^{0}$ has a noticeable peak during the gap period that is much larger in value than the variation during CHAMP or Swarm times, while the sensitivity is slightly offset to higher values. We find the same behavior for all RC-baseline corrections and calibration parameters, although most prominently for the sensitivities. Again, other model parameters seem unchanged, which indicates that there is a significant correlation between the RC-baseline corrections and the calibration parameters of the platform magnetometers. Using a single bin for the RC-baseline corrections in the gap period helps to reduce that effect. As a final comment regarding Model$\mathrm{C}$ and Model-D, we performed a simulation combining both experiments; that is, we determined $g_{1}^{0}$ with the platform magnetomter data and estimated the RC-baseline corrections in 30 day bin over the entire model time
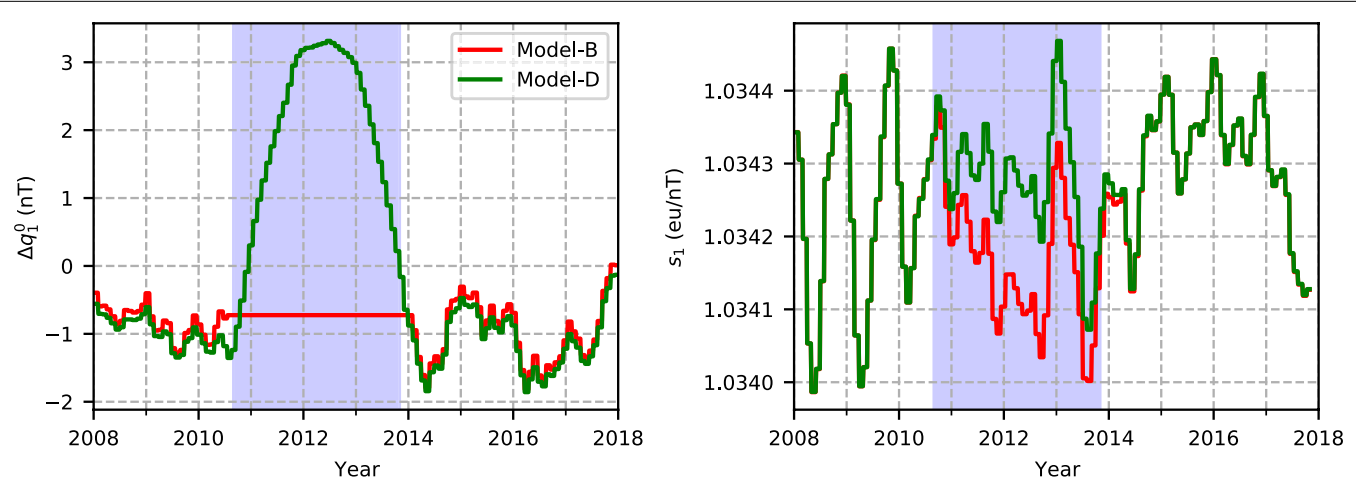

Fig. 7 Time-series of the RC-baseline correction $\Delta q_{1}^{0}$ (left) and sensitivity $s_{1}$ for GRACE-A as computed from Model-B and Model-D (right). The gap period between CHAMP and Swarm is indicated as a blue-shaded region (Sep 2010 to Nov 2013). For Model-D, the bins of the RC-baseline corrections are 30 days over the entire model time span, while they were merged to a single bin in the gap period for Model-B 
span. In this case, we observed deviations from Model-B which were identical to those shown in Figs. 6 and 7, but, now, affected the internal axial dipole, the RC-baseline corrections, and the sensitivities all at the same time.

In an effort to analyze the relationship between the calibration and the other model parameters in a quantitative manner, we also investigated the model correlations $\rho_{i j}=C_{i j} / \sqrt{C_{i i} C_{j j}}$ based on the entries of the model covariance matrix:

$$
\underline{\underline{\mathbf{C}}}=\left(\underline{\underline{\mathbf{G}}}^{\mathrm{T}} \underline{\underline{\mathbf{C}}}^{-1} \underline{\underline{\mathbf{G}}}+\underline{\underline{\Lambda}}\right)^{-1}
$$

evaluated with the converged model parameters (Tarantola 2005, p. 71). Unfortunately, the analysis revealed a large number of small correlations, which are difficult to interpret. Therefore, we did not make significant use of it in the modeling and preferred to rely on experiments to guide our modeling strategy.

In a final experiment, we derived a test model, Model$E$, by only using nightside platform magnetometer data as opposed to Model-B, where the calibration parameters were determined from dayside and nightside platform magnetometer data. Figure 8 shows the calibration parameters for GRACE-A computed with Model-B (thick lines) and Model-E (thin lines).
In the case of GRACE-A, using dayside data to determine the calibration parameters considerably changes the sensitivities and non-orthogonalities as can be seen, for example, when looking at $s_{1}, s_{2}$ or $u_{3}$. In particular for $s_{2}$, there is a vertical shift of approximately $200 \times 10^{-6} \mathrm{eu} / \mathrm{nT}$, which translates to $10 \mathrm{nT}$ in a magnetic field of $50000 \mathrm{nT}$. Irrespective of the platform magnetometer, the experiment shows that the local time coverage of the data plays an important role in determining the calibration parameters. The importance of using both day and nightside data becomes clear when appreciating that the orbital plane of the satellites is slowly drifting in local time. Under a possible nightside data selection criteria, the drift leads to the selection of data from either the ascending part or descending part of the orbit at a time. For example, if the ascending node of the orbit is on the nightside, then the platform magnetometer collects data of the magnetic field that mostly points along the direction of flight, in agreement with the predominant dipolar field configuration, until the ascending node crosses over to the dayside placing the descending part of the orbit on the nightside. Now, the observed magnetic field mostly points against the direction of flight. In the case of CryoSat-2, it takes the ascending node 8 months and GRACE around 11 months to
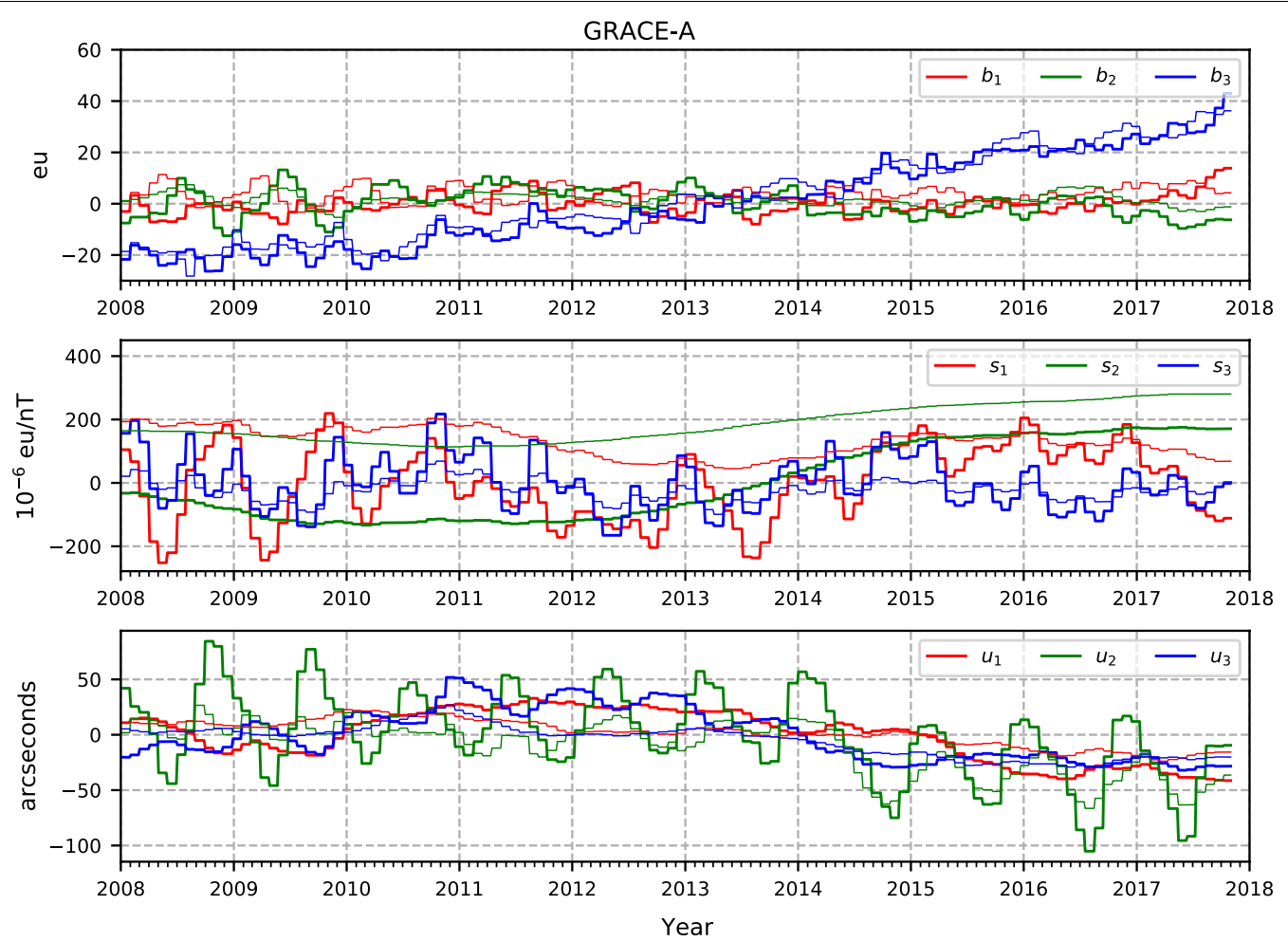

Fig. 8 Calibration parameters of GRACE-A computed with Model-B (thick lines) and Model-E (thin lines). We removed the mean values from the calibration parameters as given in Table 7 
traverse the nightside, which is longer than the monthly bins used for estimating the calibration parameters. Hence, the data of each bin will be collected either from the ascending or descending nodes with the respective bias of the field direction. Instead, using both nightside and dayside, we ensured that the data within each bin covered a broad range of local times to excite the platform magnetometer from various directions, which we believe improves the estimation of the calibration parameters. Nevertheless, we did not use any dayside data to constrain the geomagnetic field model, since we do not account for the strong ionospheric sources on the dayside. Those ionospheric sources, however, may contaminate the calibration parameters.

\section{Secular acceleration}

One motivation for using platform magnetometer data has been the growing interest in SA pulses, and enhancements of the SA that occur on sub-decadal time scales and are seen most prominently at low latitudes. These pulses have been reported by several studies (Olsen and Mandea 2007; Chulliat et al. 2010; Chulliat and Maus 2014) and are thought to reflect the dynamical processes in the Earth's outer core. To further study SA pulses and the SA in general, accurate internal field models are needed, which rely on long and continuous time-series of satellite data to give a global picture. When supplemented with high-quality satellite data, platform magnetometer data may play an important role in providing those models.

To investigate the effect of platform magnetometer data on the recovered SA, we show in Fig. 9 time-longitude maps of the radial SA on the Equator at the CMB computed with Model-B (left) and Model-A (center) alongside the difference map (right).

Recall that Model-B is partly based on platform magnetometer data in contrast to Model-A, so that the difference of the two reflects the use of these data. Both models show the SA pulses in 2009, 2013, and most recently in 2017 as enhancement of the radial SA on the Equator. Of special interest is the pulse in 2013, right in between periods of high-quality magnetic data from the CHAMP and Swarm missions. In the difference map, the SA during CHAMP and Swarm period is largely unchanged, which suggests that the effect of the CryoSat-2 and GRACE data is rather minimal during these times. In contrast, the SA in the gap period is distinctly different for the two models. Differences that are large in absolute value seem to be concentrated around $0^{\circ}$ and $180^{\circ}$ longitude on the Equator which coincides with the Pacific and the region in the South Atlantic close to Central Africa. The geographical location of the differences is more clearly seen in Fig. 10, which shows global maps of the radial SA at the CMB during the SA pulses in 2009, 2013, and 2017.

Again, the difference between Model-B and Model-A is small in 2009 and 2017, i.e., during CHAMP and Swarm times, but large in 2013 in the middle of the gap period. The regions with the largest differences are located in the Southern hemisphere and the Equatorial region with prominent examples in the West and South Pacific Ocean, and Central Africa. Our findings seem to indicate that the platform magnetometers have the desired effect of balancing the uneven spatial distribution of the ground observatory network in the gap period.
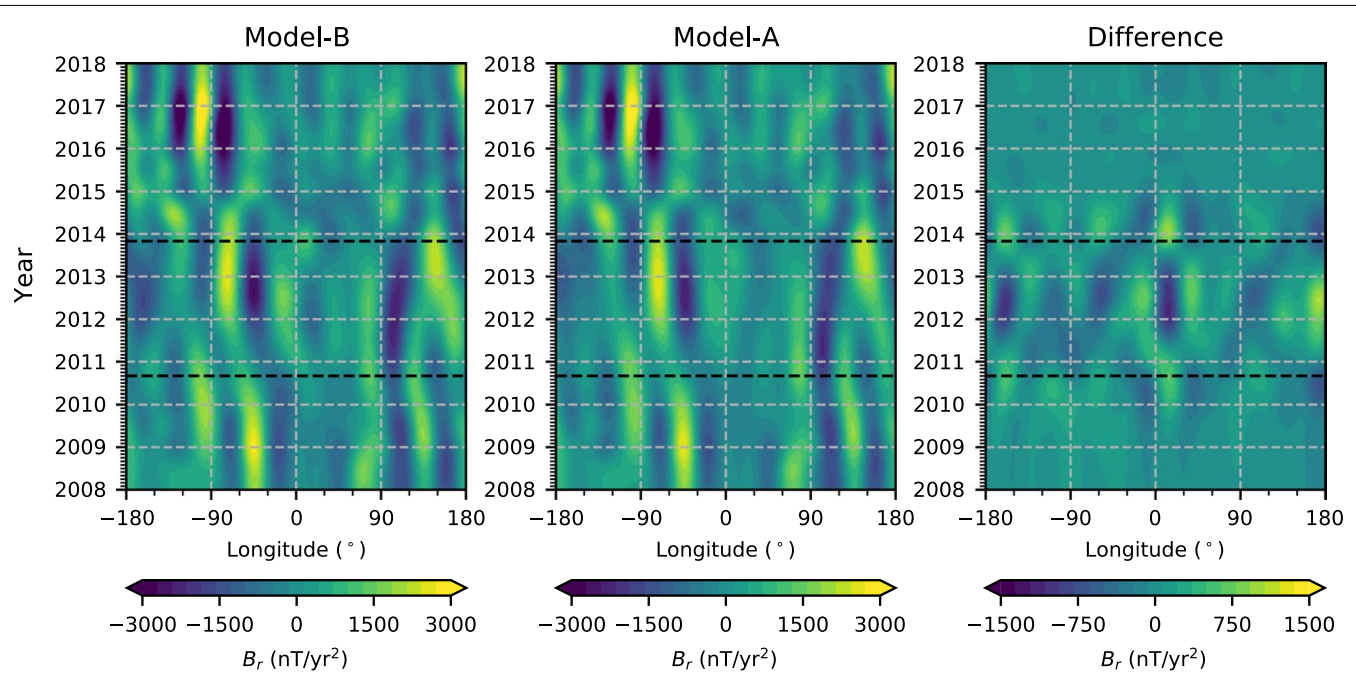

Fig. 9 Time-longitude maps of the radial SA up to degree 10 on the Equator at the CMB as computed with Model-B (left), Model-A (center) and their difference, Model-B minus Model-A (right). The gap period between CHAMP and Swarm is in between the black dashed lines (Sep 2010-Nov 2013) 


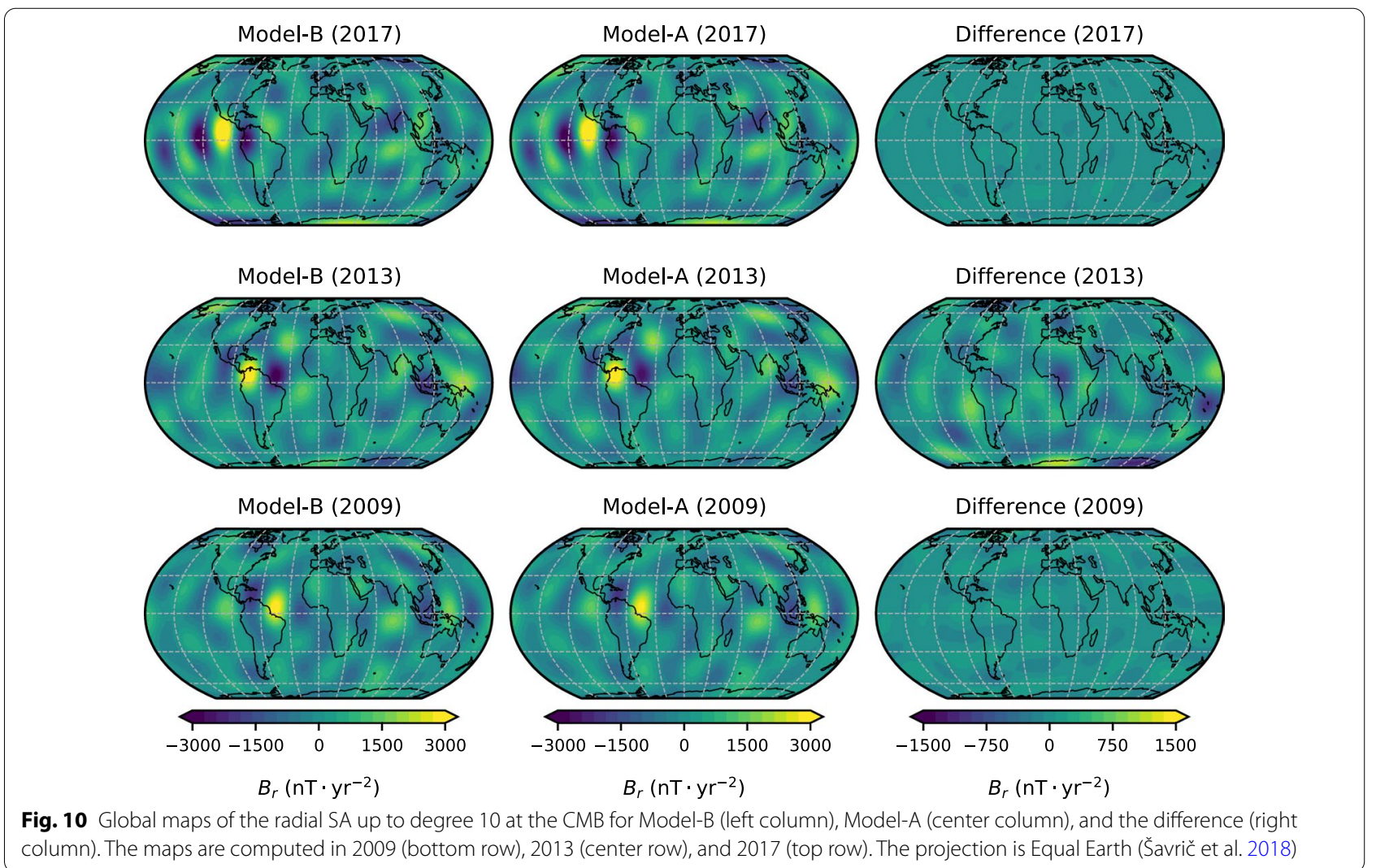

\section{Conclusions}

In this study, we present a co-estimation scheme within the framework of the CHAOS field model series that is capable of estimating both a geomagnetic field model and, at the same time, calibration parameters for platform magnetometers. This approach enables us to use platform magnetometer data to supplement high-quality magnetic data from magnetic survey satellites and removes the requirement for utilizing a-priori geomagnetic field models to calibrate platform magnetometer data.

We followed Alken et al. (2020), but went further in that we co-estimated a model of not only the internal field but also the external field. The co-estimation scheme relies on absolute magnetic data which we took from CHAMP, Swarm-A, Swarm-B, and the monthly SV data from ground observatories between 2008 and 2018. Magnetic data from five platform magnetometers were used: three on-board CryoSat-2 and one on-board each of the two GRACE satellites. This allowed us to considerably improve the geographical and temporal coverage of satellite data after CHAMP and before the launch of the Swarm satellites.

We successfully co-estimated a geomagnetic field model along with the calibration parameters of the five platform magnetometers. The misfit to the high-quality satellite data and ground observatory SV data was similar to that for models derived without including platform magnetometer data, and the good fit to an independent ground observatory dataset from Saint Helena provides evidence that our modeling approach performs well.

In a series of experiments, we investigated the tradeoffs when co-estimating calibration and geomagnetic field model parameters. We found that the calibration parameters strongly correlate with the internal axial dipole and the RC-baseline corrections of the external field during the gap period, when there is less high-quality data available. By preventing platform magnetometer data from contributing to the internal axial dipole and using constant RC-baseline corrections throughout the entire gap period, we successfully avoided those complications.

Our experiments showed that including platform magnetometer data leaves the SA signal practically unchanged during the CHAMP and Swarm period, but leads to differences in the gap period. The difference in the recovered SA signal is stronger in the West and South Pacific, where only a few observatories are located, which suggests that platform magnetometer data help to improve the global picture of the SA. Based on our investigations, we find that it is worthwhile to include platform magnetometer data in internal field modeling, in particular from CryoSat-2 given the relative low noise level. 


\begin{abstract}
Abbreviations
CHAMP: CHAllenging Minisatellite Payload; CMB: Core mantle boundary; CRF: Common reference frame; DMSP: Defense Meteorological Satellite Program; FGM: Fluxgate magnetometer; GRACE: Gravity Recovery and Climate Experiment; GSM: Geocentric solar magnetic; NEC: North-east-center; nT: NanoTesla; QD: Quasi-dipole; RTP: Radius-theta-phi; SM: Solar magnetic; VFM: Vector field magnetometer.
\end{abstract}

\section{Acknowledgements}

The European Space Agency (ESA) is gratefully acknowledged for providing access to the Swarm L1b data, CryoSat-2, and GRACE platform magnetometer data and related engineering information. We wish to thank the German Aerospace Center (DLR) and the Federal Ministry of Education and Research for supporting the CHAMP mission. Furthermore, we would like to thank the staff of the geomagnetic observatories and INTERMAGNET for supplying highquality observatory data. Susan Macmillan (BGS) is gratefully acknowledged for collating checked and corrected observatory hourly mean values in the AUX OBS database.

\section{Open Access}

This article is distributed under the terms of the Creative Commons Attribution 4.0 International License (http://creativecommons.org/licenses/by/4.0/), which permits unrestricted use, distribution, and reproduction in any medium, provided you give appropriate credit to the original author(s) and the source, provide a link to the Creative Commons license, and indicate if changes were made.

\section{Authors' contributions}

CK developed the modeling software for the co-estimation of calibration parameters, derived the presented models, and led the writing of the manuscript. CCF participated in the design of the study. NiO pre-processed the CryoSat-2 and GRACE platform magnetometer data, developed the CHAOS modeling approach, and participated in the design of the study. All authors read and approved the final manuscript.

\section{Funding}

CK and CCF were funded by the European Research Council (ERC) under the European Union's Horizon 2020 research and innovation programme (grant agreement No. 772561). The study has been partly supported as part of Swarm DISC activities, funded by ESA contract no. 4000109587.

\section{Availability of data and materials}

The datasets supporting the conclusions of this article are available in the following repositories: Swarm and CryoSat-2 data are available from https:// earth.esa.int/web/guest/swarm/data-access; The GRACE data are available from ftp://ftp.spacecenter.dk/data/magnetic-satellites/GRACE/; CHAMP data are available from https://isdc.gfz-potsdam.de/champ-isdc; Ground observatory data are available from ftp://ftp.nerc-murchison.ac.uk/geomag/Swarm/ AUX_OBS/hour/; The RC-index is available from http://www.spacecenter.dk/ files/magnetic-models/RC/; The CHAOS-6 model and its updates are available from http://www.spacecenter.dk/files/magnetic-models/CHAOS-6/; and Solar wind speed, interplanetary magnetic field, and $\mathrm{Kp}$-index are available from https://omniweb.gsfc.nasa.gov/ow.html.

\section{Competing interests}

The authors declare that they have no competing interests.

Received: 30 September 2020 Accepted: 26 December 2020

Published online: 20 January 2021

\section{References}

Alken P, Olsen N, Finlay CC (2020) Co-estimation of geomagnetic field and inorbit fluxgate magnetometer calibration parameters. Earth Planets Space 72(1):1-32. https://doi.org/10.1186/s40623-020-01163-9

Backus G, George B, Parker RL, Parker R, Constable C (1996) Foundations of Geomagnetism. Cambridge University Press, Cambridge
Chulliat A, Maus S (2014) Geomagnetic secular acceleration, jerks, and a localized standing wave at the core surface from 2000 to 2010. J Geophys Res 119(3):1531-1543. https://doi.org/10.1002/2013jb010604

Chulliat A, Thebault E, Hulot G (2010) Core field acceleration pulse as a common cause of the 2003 and 2007 geomagnetic jerks. Geophys Res Lett 37:7. https://doi.org/10.1029/2009gl042019

Constable CG (1988) Parameter estimation in non-gaussian noise. Geophys J Int 94(1):131-142

De Boor C (1978) A practical guide to splines, vol 27. Springer, New York

Finlay CC, Olsen N, Kotsiaros S, Gillet N, Tøffner-Clausen L (2016) Recent geomagnetic secular variation from Swarm and ground observatories as estimated in the CHAOS-6 geomagnetic field model. Earth Planets Space 68:1. https://doi.org/10.1186/s40623-016-0486-1

Finlay CC, Kloss C, Olsen N, Hammer M, Tøffner-Clausen L, Grayver A, Kuvshinov A (2020) The CHAOS-7 geomagnetic field model and observed changes in the south atlantic anomaly. Earth Planets Space. https://doi. org/10.1186/s40623-020-01252-9

Holme R, Bloxham J (1996) The treatment of attitude errors in satellite geomagnetic data. Phys Earth Planet Inter 98(3-4):221-233

Macmillan S, Olsen N (2013) Observatory data and the Swarm mission. Earth Planets Space 65(11):1355-1362. https://doi.org/10.5047/eps.2013.07.011

Olsen N (2020) Magnetometer data of the GRACE satellites duo. Earth Planets Space (in review)

Olsen N, Mandea M (2007) Investigation of a secular variation impulse using satellite data: The 2003 geomagnetic jerk. Earth Planet Sci Lett 255(1-2):94-105. https://doi.org/10.1016/j.epsl.2006.12.008

Olsen $\mathrm{N}$ et al (2003) Calibration of the Ørsted vector magnetometer. Earth Planets Space 55(1):11-18. https://doi.org/10.1186/BF03352458

Olsen N, Lühr H, Sabaka TJ, Mandea M, Rother M, Tøffner-Clausen L, Choi S (2006) CHAOS-a model of the Earth's magnetic field derived from CHAMP, Ørsted, and SAC-C magnetic satellite data. Geophys J Int 166(1):67-75. https://doi.org/10.1111/j.1365-246x.2006.02959.x

Olsen N, Lühr H, Finlay CC, Sabaka TJ, Michaelis I, Rauberg J, Tøffner-Clausen $\mathrm{L}$ (2014) The CHAOS-4 geomagnetic field model. Geophys J Int 197(2):815-827

Olsen N, Albini G, Bouffard J, Parrinello T, Tøffner-Clausen L (2020) Magnetic observations from CryoSat-2: calibration and processing of satellite platform magnetometer data. Earth Planets Space 72:1-18

Richmond AD (1995) lonospheric electrodynamics using magnetic apex coordinates. J Geomagn Geoelectr 47(2):191-212. https://doi.org/10.5636/ jgg.47.191

Rother, M., and I. Michaelis (2019), CH-ME-3-MAG - CHAMP 1 Hz combined magnetic field time series (level 3), https://doi.org/10.5880/ GFZ.2.3.2019.004

Sabaka TJ, Olsen N, Purucker ME (2004) Extending comprehensive models of the earth's magnetic field with ørsted and CHAMP data. Geophys J Int 159(2):521-547

Sabaka, T. J., G. Hulot, and N. Olsen (2010), Mathematical properties relevant to geomagnetic field modeling. In: Handbook of geomathematics. Springer: New York. pp. 503-538

Šavrič B, Patterson T, Jenny B (2018) The equal earth map projection. Int J Geogr Inform Sci 33(3):454-465. https://doi.org/10.1080/13658 816.2018 .1504949

Tarantola A (2005), Inverse problem theory and methods for model parameter estimation, SIAM

Thébault E et al (2015) International geomagnetic reference field: the 12th generation. Earth Planets Space 67(1):79

\section{Publisher's Note}

Springer Nature remains neutral with regard to jurisdictional claims in published maps and institutional affiliations. 